\title{
Intersection de sous-groupes et de sous-variétés III
}

\author{
Gaël Rémond
}

\begin{abstract}
We elucidate the structure of various exceptional subsets appearing in parts I and II in order to prove new results on the Zilber-Pink conjecture for abelian varieties. In particular, we obtain boundedness of height on the intersection of interest for all non-degenerate varieties (in a precise sense). The main idea to prove that our exceptional subsets are closed comes from a result of Bombieri, Masser and Zannier on tori and we follow the same approach through so-called anomalous subvarieties but we have to allow extra generality in the definition. We also use in a crucial way a theorem of Ax on analytic subgroups of algebraic groups.
\end{abstract}

Mathematics Subject Classification (2000). 11G10, 11G50, 14K12.

Mots-Clefs. Variétés abéliennes, conjecture de Zilber-Pink.

\section{Introduction}

En avril 2005, R. Pink a formulé une conjecture sur les variétés de Shimura mixtes qui se spécialise en l'énoncé suivant sur les variétés abéliennes (voir [P]).

Conjecture 1.1. Soient $A$ une variété abélienne sur un corps $K$ de caractéristique zéro et $X$ un sous-schéma fermé intègre de $A$ qui n'est contenu dans aucun sous-schéma en groupes de $A$ différent de $A$. Alors l'ensemble

$$
X(K) \cap \bigcup_{\operatorname{dim} X+\operatorname{dim} G<\operatorname{dim} A} G(K),
$$

où l'union porte sur les sous-schémas en groupes $G$ de $A$ vérifiant la condition de dimension, n'est pas dense dans $X$.

Ceci constitue une version de la conjecture de Zilber-Pink sur les variétés semiabéliennes. Pour la version due antérieurement à Zilber (2002), on consultera [Z] ainsi que [BMZ] où les auteurs examinent les liens entre ces conjectures et de nouvelles variantes qui leur sont dues (2006). En particulier, les formulations de Zilber et de Pink sont équivalentes dans le cas des tores. Il convient de préciser que cette famille de problèmes trouve son origine dans un travail de E. Bombieri, D. Masser et U. Zannier 
de 1999 sur les tores qui prédate donc toutes les conjectures (voir les références de [BMZ]).

Dans les parties I et II de cette série d'articles, nous avons donné des résultats partiels en direction de la conjecture 1.1 faisant intervenir divers ensembles exceptionnels à enlever à $X$ (voir [R1] et [R2]). Nous précisons ici ces résultats en élucidant les liens entre ces ensembles et en montrant qu'un certain nombre d'entre eux sont fermés. Pour les autres faits connus sur ce problème, on se reportera à [BMZ] et [Ma] pour les tores ainsi qu'à [Rat] pour les courbes dans les variétés abéliennes.

Pour énoncer nos résultats, nous introduisons quelques notations. Nous fixons $A$ et $K$ comme dans la conjecture 1.1. Pour tout entier $r$ avec $0 \leq r \leq \operatorname{dim} A$, nous écrivons comme dans [R1]

$$
A^{[r]}=\bigcup_{\operatorname{codim} G \geq r} G(K)
$$

où $G$ parcourt tous les sous-schémas en groupes de $A$ de codimension au moins $r$. La conjecture ci-dessus concerne donc $X(K) \cap A^{[\mathrm{dim} X+1]}$. Il se trouve qu'elle est équivalente à la variante suivante avec un groupe $\Gamma$ (voir $[\mathrm{P}])$.

Conjecture 1.2. Soient $X$ un sous-schéma fermé intègre de $A$ qui n'est contenu dans aucun translaté de sous-variété abélienne de $A$ différente de $A$ et $\Gamma$ un sous-groupe de rang fini de $A(K)$. Alors $X(K) \cap\left(\Gamma+A^{\text {[dim } X+1]}\right)$ n'est pas dense dans $X$.

Tous les résultats connus à ce jour en direction de cette conjecture imposent une condition plus forte sur $X$ (pour cette terminologie voir [Ran] et [D]).

Définition 1.1. On dit que $X$ est géométriquement non dégénéré si pour toute sousvariété abélienne $B$ de $A$ on a $\operatorname{dim}(X+B)=\min (\operatorname{dim} X+\operatorname{dim} B, \operatorname{dim} A)$.

Nous verrons aussi ci-dessous qu'il suffit de vérifier cette égalité pour un ensemble fini de sous-variétés abéliennes $B$ dépendant seulement du degré de $X$ (voir proposition 4.2).

Ceci nous permet d'énoncer le résultat suivant (démontré à la fin de la partie 4), qui découle de l'étude des ensembles exceptionnels et d'un résultat récent de M. Carrizosa en direction du problème de Lehmer sur les variétés abéliennes (voir [C]).

Théorème 1.3. Soient A une variété abélienne à multiplications complexes sur $K=$ $\overline{\mathbb{Q}}, X$ un sous-schéma fermé intègre de A géométriquement non dégénéré et $\Gamma$ un sous-groupe de rang fini de $A(K)$. Alors $X(K) \cap\left(\Gamma+A^{[\mathrm{dim} X+1]}\right)$ n'est pas dense dans $X$.

Signalons que $\mathrm{P}$. Habegger [Hab] obtient ce théorème dans le cas particulier où $\Gamma=0$ par des méthodes différentes de celles que nous présentons ici (même s'il y a plusieurs points de contact, comme l'application du théorème d'Ax, voir plus bas). 
Pour présenter les ingrédients intervenant dans le théorème 1.3, nous revenons sur les ensembles définis dans les parties I et II. Tout d'abord, pour un sous-groupe $\Gamma$ de $A(K)$, nous notons $\Gamma_{\text {sat }}$ le groupe de division de $\operatorname{End}(A) \cdot \Gamma$ puis, pour un entier $r$ et un sous-schéma fermé intègre $X$ de $A$, nous introduisons la partie $Z_{X, \Gamma}^{(r)}$ de $X(K)$ formée des points $x$ pour lesquels il existe $\gamma \in \Gamma_{\text {sat }}$ et une sous-variété abélienne $B$ de $A$ tels que

$$
\operatorname{dim}_{x} X \cap(\gamma+B)>\max (0, r-1-\operatorname{codim} B) .
$$

Cet ensemble intervient dans [R1] (voir page 531). Ici nous utilisons surtout l'union $Z_{X \text {,an }}^{(r)}$ des $Z_{X, \Gamma}^{(r)}$ pour tous les sous-groupes $\Gamma$ qui peut être définie plus directement ainsi.

Définition 1.2. Soit $Z_{X \text {,an }}^{(r)}$ la partie de $X(K)$ formée des points $x$ pour lesquels il existe une sous-variété abélienne $B$ de $A$ telle que

$$
\operatorname{dim}_{x} X \cap(x+B)>\max (0, r-1-\operatorname{codim} B) .
$$

Cet ensemble coïncide avec $X \backslash X^{\text {oa }}$ dans les notations de [BMZ] lorsque $r=$ $\operatorname{dim} X+1$. Nous le verrons aussi comme union de sous-variétés anormales de $X$ (ceci explique l'indice dans la notation $Z_{X \text {,an }}^{(r)}$ de même que oa rappelle open anomalous).

Ensuite, deux ensembles $Z_{X, \mathbb{Q}}^{(r)}$ et $Z_{X}^{(r)}$ sont étudiés dans [R2] (voir p. 319). Pour rappeler leur définition, nous notons $Z_{+}^{1}(A)$ le cône des diviseurs effectifs sur $A$ à coefficients réels modulo équivalence numérique. C'est une partie de $\mathrm{NS}(A) \otimes \mathbb{R}$ où $\mathrm{NS}(A)$ est le groupe de Néron-Severi de $A$. Nous disons qu'un fermé irréductible $Y$ de $X$ est $r$-supertransverse si :

pour tous $\mathcal{N}, \mathcal{N}_{1}, \mathcal{N}_{2} \in Z_{+}^{1}(A)$ et $a \in \mathbb{N}$ vérifiant $\operatorname{rg} \mathcal{N} \geq r, \mathcal{N}=\mathcal{N}_{1}+\mathcal{N}_{2}$ et $\operatorname{rg} \mathcal{N}_{1}-1 \leq a \leq \operatorname{dim} Y$, on a $\mathcal{N}^{\cdot a} \cdot \mathcal{N}_{2}^{\cdot \operatorname{dim} Y-a} \cdot Y>0$.

De même $Y$ est dit $r$ - $\mathbb{Q}$-supertransverse si cette condition vaut pour tous les éléments $\mathcal{N}, \mathcal{N}_{1}, \mathcal{N}_{2} \in Z_{+}^{1}(A) \cap(\mathrm{NS}(A) \otimes \mathbb{Q})$.

Définition 1.3. On note $Z_{X}^{(r)}$ l'union des fermés irréductibles de $X$ qui ne sont pas $r$-supertransverses et $Z_{X, \mathbb{Q}}^{(r)}$ l'union de ceux qui ne sont pas $r$ - $\mathbb{Q}$-supertransverses.

Dans [R2] le résultat principal montrait que l'intersection $\left(X(\overline{\mathbb{Q}}) \backslash Z_{X}^{(r)}\right) \cap(\Gamma+$ $\left.A^{[r]}\right)$ est toujours de hauteur bornée mais la question de la nature de $Z_{X}^{(r)}$ restait en suspens. Notre théorème principal y répond et relie les trois ensembles exceptionnels apparus jusqu'ici. 
Théorème 1.4. Pour tout entier $r$ avec $0 \leq r \leq \operatorname{dim} A$, nous avons

$$
Z_{X, \mathbb{Q}}^{(r)} \subset Z_{X}^{(r)} \subset Z_{X, \text { an }}^{(r)}
$$

et ces trois ensembles sont fermés.

Lorsque $A$ est isogène à un produit de courbes elliptiques, les trois fermés coïncident mais en général nous donnerons des exemples montrant que chacune des inclusions peut être stricte. La preuve de la fermeture s'inspire de [BMZ] où est établi l'analogue torique du fait que $Z_{X \text {,an }}^{(r)}$ est fermé pour $r=\operatorname{dim} X+1$. La partie la plus nouvelle ici concerne l'inclusion $Z_{X}^{(r)} \subset Z_{X \text {,an }}^{(r)}$. Elle permet d'exploiter le théorème de [R2] pour obtenir le théorème 1.3.

Nous verrons aussi comme simple corollaire de la structure de ces ensembles exceptionnels le critère suivant (démontré sous la forme plus précise de la proposition 4.2 à la fin de la partie 4).

Théorème 1.5. Le fermé $X$ est géométriquement non dégénéré si et seulement si $Z_{X, \text { an }}^{(\operatorname{dim} X+1)} \neq X$.

Pour aller plus loin dans la direction de la conjecture de Zilber-Pink, il faudrait savoir répondre aux deux questions suivantes.

Question 1. L'ensemble $\left(X(K) \backslash Z_{X, \Gamma}^{(r)}\right) \cap\left(\Gamma+A^{[r]}\right)$ est-il fini ?

Question 2. L'ensemble $Z_{X, \Gamma}^{(r)}$ est-il fermé ?

Pour $\Gamma=0$, la question 1 était posée dans [R1]. Pour $\Gamma=0$ et $r=\operatorname{dim} X+1$, ces deux questions correspondent aux conjectures dites respectivement torsion-finiteness et torsion-openness dans [BMZ]. Ils montrent qu'ensemble elles entraînent (sur $\mathbb{C}$ ) la conjecture 1.1 pour les tores.

Nous montrons ici (voir partie 6) que la question 1 implique la question 2. On notera que pour les tores (avec $\Gamma=0, r=\operatorname{dim} X+1$ et $K=\mathbb{C}$ ) la réciproque est montrée dans [BMZ] (en laissant varier $A$ ). Pour répondre positivement à la question 2, il faut montrer que $X$ possède un nombre fini de sous-variétés $\Gamma$-anormales maximales (terminologie analogue à celle de [BMZ], voir partie 6). Comme premier pas, nous montrons inconditionnellement que ces sous-variétés sont de degré borné.

Les trois parties suivantes sont consacrées à la démonstration du théorème 1.4. L'idée consiste à écrire les trois ensembles $Z_{X, \mathbb{Q}}^{(r)}, Z_{X}^{(r)}$ et $Z_{X \text {,an }}^{(r)}$ sous une forme commune à savoir

$$
Z_{X}(\mu)=\left\{x \in X(K) \mid \exists \varphi \in \operatorname{End}(A) \operatorname{dim}_{x} \varphi^{-1}(\varphi(x)) \cap X>\mu(\varphi)\right\}
$$

pour une certaine fonction $\mu: \operatorname{End}(A) \rightarrow \mathbb{N}$ bien choisie (les définitions de fonctions $\mu$ convenables seront données au début de la partie 4). Nous montrons d'abord 
qu'un tel ensemble est fermé en adaptant la méthode de [BMZ] : c'est le lieu où certaines fibres ont une dimension anormale, nous parlons de fermé $\mu$-anormal et nous montrons que les fermés $\mu$-anormaux maximaux sont de degré borné. Ceci fait l'objet de la partie 3 et se base sur un critère différentiel de dimension des fibres que nous examinons en partie 2. Il nous faut faire apparaître non seulement les endomorphismes de $A$ mais aussi ceux de son espace tangent, ce qui implique des arguments analytiques. Pour cela nous travaillons sur $\mathbb{C}$ mais les résultats sont géométriques et se transportent à $\overline{\mathbb{Q}}$.

Ensuite, dans la partie 4, nous identifions les ensembles de départ avec des $Z_{X}(\mu)$. C'est immédiat pour $Z_{X \text {,an }}^{(r)}$, facile pour $Z_{X, \mathbb{Q}}^{(r)}$ mais demande du travail pour $Z_{X}^{(r)}$. Là encore des arguments analytiques entrent en jeu et nous utilisons un théorème d' $\mathrm{Ax}$ (voir $[\mathrm{Ax}])$. Enfin la partie 5 présente des exemples où nos différents ensembles sont effectivement distincts et nous concluons en partie 6 avec les résultats concernant les questions 1 et 2 ci-dessus.

Expliquons l'idée de la stratégie suivie dans les parties 2 et 3 ci-dessous. Pour simplifier, imaginons dans un premier temps qu'une fibre $Y=X \cap \varphi^{-1}(\varphi(x))$ soit irréductible. Nous pouvons dans cette situation contrôler facilement le degré de $Y$ en fonction de celui du noyau de $\varphi$ (par le théorème de Bézout) et réciproquement, étant donné $Y$, il est possible de trouver un endomorphisme $\varphi$ dont le noyau est de degré contrôlé en fonction de deg $Y$ (c'est un peu moins direct : il faut considérer la plus petite sous-variété abélienne dont un translaté contient $Y$, voir lemme 2.6). Cette réciproque montre que, si le degré des fibres de dimension anormale était borné par une constante, nous pourrions nous contenter d'un nombre fini de $\varphi$ et donc $Z_{X}(\mu)$ serait fermé. C'est essentiellement ce que nous ferons ; toutefois ceci ne s'applique pas à toutes les telles fibres mais seulement à celles qui ne sont pas contenues dans une autre fibre anormale (pour un autre morphisme $\psi$ ). L'idée principale consiste à estimer le degré de $Y$ non pas directement par le théorème de Bézout, ce qui revient à couper $X$ par des équations définissant $\varphi$, mais plutôt à utiliser des équations définissant la différentielle de $\varphi$ (pour une notion formelle, voir définition 2.2). Ceci présente l'intérêt que, tandis que le degré d'équations de $\varphi$ croît avec le degré du noyau de $\varphi$, pour la différentielle on peut utiliser un degré constant (ceci apparaît dans le lemme 2.5). Le revers de la médaille est qu'en général les équations obtenues définissent un fermé plus grand que $Y$, même lorsque $Y$ est maximal parmi les fibres anormales, et ceci oblige à travailler par récurrence sur la dimension de $X$. De plus, le critère différentiel esquissé ici n'est vraiment efficace que lorsque le point $\varphi(x)=\varphi(Y)$ est lisse dans $\varphi(X)$. Pour contourner cet obstacle plusieurs contorsions sont nécessaires (voir lemme 2.4, lemme 3.3 et la fin de l'argument) et il faut aussi tenir compte du fait que $Y$ n'est pas irréductible mais peut contenir plusieurs composantes de dimensions différentes.

Je tiens à remercier ici E. Bombieri, D. Masser et U. Zannier de m'avoir communiqué leur texte [BMZ] avant publication. 


\section{Critère jacobien}

Nous considérons une variété abélienne complexe $A$ de dimension $g$ que nous écrivons $A=V / \Lambda$ où $V$ est l'espace tangent à l'origine de $A$ (un $\mathbb{C}$-espace vectoriel de dimension $g$ ) et $\Lambda$ un réseau de $V$. Nous notons $\pi: V \rightarrow V / \Lambda$ la projection et $\operatorname{End}(V)$ les endomorphismes de l'espace vectoriel $V$.

Nous rappelons que l'anneau $\operatorname{End}(A)$ des endomorphismes de $A$ s'identifie au sous-anneau $\{\varphi \in \operatorname{End}(V) \mid \varphi(\Lambda) \subset \Lambda\}$ de $\operatorname{End}(V)$. Ceci crée une ambiguité pour parler du noyau de $\varphi \in \operatorname{End}(A)$; afin de la lever, nous désignerons toujours par $\operatorname{Ker} \varphi \subset V$ son noyau comme endomorphisme de $V$ tandis que nous utiliserons la notation $\operatorname{Ker}^{0} \varphi$ pour nous référer à la composante neutre du noyau de $\varphi$ comme endomorphisme de $A$. La formule $\operatorname{Ker}^{0} \varphi=\operatorname{Ker} \varphi / \Lambda \cap \operatorname{Ker} \varphi$ fait le lien entre les deux conventions.

Définition 2.1. Soient $X$ une sous-variété algébrique de $A$ et $\tilde{X}=\pi^{-1}(X)$. Pour $\varphi \in \operatorname{End}(V)$ nous notons $\operatorname{dim} \varphi(X)$ l'entier

$$
\sup _{y \in \tilde{X}}\left(\operatorname{dim} X-\operatorname{dim}_{y} \tilde{X} \cap(y+\operatorname{Ker} \varphi)\right) .
$$

Notons que $\tilde{X}$ est un sous-ensemble analytique de $V$ et qu'il en va donc de même de $\tilde{X} \cap(y+\operatorname{Ker} \varphi)$ ce qui donne un sens à la définition. Intuitivement, ce nombre devrait être la dimension de $\varphi(\tilde{X})$ mais cet ensemble n'est pas en général analytique (ni même fermé). Toutefois il existe un ouvert dense $\widetilde{X}^{\prime}$ de $\widetilde{X}$ tel que tout point $y$ de $\widetilde{X}^{\prime}$ admette un voisinage $U$ dans $\tilde{X}^{\prime}$ de sorte que $\varphi(U)$ soit une sous-variété analytique d'un ouvert de $V$ de dimension égale à l'entier $\operatorname{dim} \varphi(X)$ de notre définition. En outre, lorsque $\varphi \in \operatorname{End}(A) \subset \operatorname{End}(V)$, la notation $\varphi(X)$ a un sens et nous retrouvons bien la dimension de cette variété algébrique. Notre convention étend donc cette notation usuelle à un cas plus général.

Notre première tâche consiste à calculer cet entier $\operatorname{dim} \varphi(X)$ en termes différentiels. Pour cela, nous considérons d'une part la suite exacte

$$
\mathcal{I}_{X} /\left.\mathcal{I}_{X}^{2} \stackrel{\delta}{\longrightarrow}\left(\Omega_{A / \mathbb{C}}^{1}\right)\right|_{X} \longrightarrow \Omega_{X / \mathbb{C}}^{1} \longrightarrow 0
$$

où $\mathcal{I}_{X}$ est le faisceau d'idéaux de $X$ dans $\mathcal{O}_{A}$. D'autre part, comme $V$ s'identifie au dual de $\Gamma\left(A, \Omega_{A / \mathbb{C}}^{1}\right)$, un élément $\varphi \in \operatorname{End}(V)$ induit un morphisme ${ }^{t} \varphi: \Gamma\left(A, \Omega_{A / \mathbb{C}}^{1}\right) \rightarrow$ $\Gamma\left(A, \Omega_{A / \mathbb{C}}^{1}\right)$ et, $\Omega_{A / \mathbb{C}}^{1}$ étant libre, un morphisme de faisceaux $\Omega_{A / \mathbb{C}}^{1} \rightarrow \Omega_{A / \mathbb{C}}^{1}$ que nous notons encore ${ }^{t} \varphi$.

Définition 2.2. Soient $\varphi \in \operatorname{End}(V)$ et $Y$ un sous-schéma fermé intègre de $X$ de point générique $\eta$. Nous notons $r(X, Y, \varphi)=\operatorname{dim}_{\kappa(\eta)} \operatorname{Im}\left(\delta_{\eta} \otimes \kappa(\eta)\right)+\left(\operatorname{Im}^{t} \varphi\right)_{\eta} \otimes \kappa(\eta)$. 
Ici $\kappa(\eta)$ désigne le corps résiduel au point $\eta$ autrement dit le corps des fonctions de $Y$ tandis que $\delta_{\eta}$ est le morphisme de $\mathcal{O}_{X, \eta}$-modules déduit de $\delta$. On notera que $\operatorname{Im}\left(\delta_{\eta} \otimes \kappa(\eta)\right)$ diffère en général de $(\operatorname{Im} \delta)_{\eta} \otimes \kappa(\eta)$, la surjection naturelle du premier vers le second n'étant pas nécessairement injective. Nous obtenons un entier au plus égal à $g$ puisque $\operatorname{Im}\left(\delta_{\eta} \otimes \kappa(\eta)\right)$ et $\left(\operatorname{Im}^{t} \varphi\right)_{\eta} \otimes \kappa(\eta)$ sont deux sous-espaces vectoriels de $\left(\Omega_{A / \mathbb{C}}^{1}\right)_{\eta} \otimes \kappa(\eta) \simeq \kappa(\eta)^{g}$.

Voici le lien entre nos définitions.

Lemme 2.1. Pour tous $X$ et $\varphi \in \operatorname{End}(V)$ nous avons

$$
\operatorname{dim} \varphi(X)=r(X, X, \varphi)+\operatorname{dim} X-g .
$$

Démonstration. Au point générique $\xi$ de $X$, nous avons $\mathcal{O}_{X, \xi}=\kappa(\xi)$ donc $\operatorname{Im}\left(\delta_{\xi} \otimes\right.$ $\kappa(\xi))$ s'identifie à $(\operatorname{Im} \delta)_{\xi}$. Ceci entraîne que, si nous choisissons un ouvert pour la topologie de Zariski $U_{0}$ de $X$, non vide, lisse et tel que les faisceaux $\left.(\operatorname{Im} \delta)\right|_{U_{0}}$ et $\left.(\operatorname{Im} \delta)\right|_{U_{0}}+\left.\left(\operatorname{Im}^{t} \varphi\right)\right|_{U_{0}}$ soient libres, alors le rang du second est $r(X, X, \varphi)$. Ensuite nous considérons un ouvert analytique non vide $U_{1}$ de $U_{0}$ suffisamment petit pour pouvoir le relever en un ouvert isomorphe $\widetilde{U}$ de $\widetilde{X}$ tel que $\varphi(\widetilde{U})$ soit une variété analytique non singulière de dimension $\operatorname{dim} \varphi(X)$. Nous avons donc un diagramme

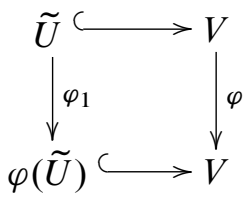

qui donne naissance en termes de faisceaux analytiques à un autre diagramme à lignes exactes

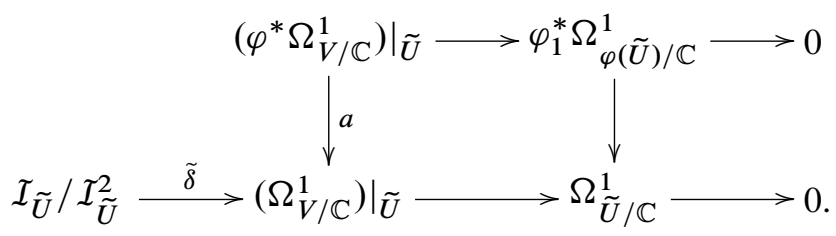

Quitte à restreindre encore $\widetilde{U}$, nous pouvons supposer que la différentielle de $\varphi_{1}$ est surjective, autrement dit que l'application $\varphi_{1}^{*} \Omega_{\varphi(\widetilde{U}) / \mathbb{C}}^{1} \rightarrow \Omega_{\widetilde{U} / \mathbb{C}}^{1}$ est injective. L'image de cette application a donc pour $\operatorname{rang} \operatorname{dim} \varphi(\widetilde{U})=\operatorname{dim} \varphi(X)$ par lissité. Le diagramme montre que cette image est aussi celle de $\left.\left(\varphi^{*} \Omega_{U / \mathbb{C}}^{1}\right)\right|_{\widetilde{U}} \rightarrow \Omega_{\widetilde{U} / \mathbb{C}}^{1}$ qui s'écrit $(\operatorname{Im} a+\operatorname{Im} \tilde{\delta}) / \operatorname{Im} \tilde{\delta}$. Il vient

$$
\operatorname{dim} \varphi(X)=\operatorname{rang}(\operatorname{Im} a+\operatorname{Im} \tilde{\delta})-\operatorname{rang}(\operatorname{Im} \tilde{\delta}) .
$$

Par lissité de $U_{0}(\operatorname{donc} \operatorname{de} \tilde{U})$ nous avons $\operatorname{rang}(\operatorname{Im} \tilde{\delta})=g-\operatorname{dim} X$. Par ailleurs $\operatorname{Im} a+$ $\operatorname{Im} \tilde{\delta}$ est la restriction à $U_{1} \simeq \widetilde{U}$ de l'analytifié du faisceau $\left.(\operatorname{Im} \delta)\right|_{U_{0}}+\left.\left(\operatorname{Im}^{t} \varphi\right)\right|_{U_{0}}$ donc il est de rang $r(X, X, \varphi)$. Ceci conclut. 
On rapprochera cet énoncé du lemme 1 de [BMZ]. L'entier $r(X, Y, \varphi)$ est l'analogue de la quantité $\operatorname{rang}_{y} \mathcal{S}\left(z_{1}, \ldots, z_{h} ; \mathcal{X}\right)$ utilisée dans cet article et notre résultat montre en particulier que si $r(X, X, \varphi) \leq g-\operatorname{dim} X+h-1$ alors $\operatorname{dim} \varphi(X) \leq h-1$.

La preuve que nous donnons dans la partie suivante joue notamment entre les propriétés vraies pour $\varphi \in \operatorname{End}(V)$ et celles uniquement valables pour $\varphi \in \operatorname{End}(A)$. Il est toutefois un cas où la différence n'a pas lieu d'être.

Lemme 2.2. Si $\varphi \in \operatorname{End}(V)$ vérifie $\operatorname{dim} \varphi(X)=0$ alors il existe une factorisation $\varphi=\chi \circ \psi$ avec $\chi \in \operatorname{End}(V)$ et $\psi \in \operatorname{End}(A)$ telle que $\operatorname{dim} \psi(X)=0$.

Démonstration. L'hypothèse montre que toute composante de $\tilde{X}$ est contenue dans un translaté de $\operatorname{Ker} \varphi$. Par connexité de $X$ on en déduit qu'il existe $y \in \tilde{X}$ tel que $\tilde{X} \subset$ $y+\Lambda+\operatorname{Ker} \varphi$. Comme $\Lambda+\operatorname{Ker} \varphi$ est un groupe il vient $\tilde{X}+\cdots+\tilde{X} \subset g y+\Lambda+\operatorname{Ker} \varphi$ où l'on fait la somme de $g$ copies de $\tilde{X}$. Par ailleurs $\tilde{X}+\cdots+\tilde{X}=\pi^{-1}(X+\cdots+X)$ et $X+\cdots+X$ est un translaté de sous-variété abélienne de $A$, disons $x+W /(W \cap \Lambda)$ où $W$ est un sous-espace vectoriel de $V$ tel que $W \cap \Lambda$ est un réseau de $W$. Par suite $\tilde{X}+\cdots+\tilde{X}$ est de la forme $y^{\prime}+W$ ce qui donne $W \subset \Lambda+\operatorname{Ker} \varphi$ puis, $\Lambda$ étant discret, $W \subset \operatorname{Ker} \varphi$. Nous choisissons $\psi \in \operatorname{End}(A)$ avec $\operatorname{Ker}^{0} \psi=W / W \cap \Lambda$ ce qui force $\operatorname{Ker} \psi=W$. Ainsi nous avons $\tilde{X} \subset y+W=y+\operatorname{Ker} \psi \operatorname{donc} \operatorname{dim} \psi(X)=0$ et l'inclusion $\operatorname{Ker} \psi=W \subset \operatorname{Ker} \varphi$ montre l'existence d'une factorisation $\varphi=$ $\chi \circ \psi$.

Nous étudions maintenant l'entier $r(X, Y, \varphi)$ pour $Y \neq X$. La première relation est évidente.

Lemme 2.3. Pour $Y$ sous-schéma fermé intègre de $X$ on a $r(X, Y, \varphi) \leq r(Y, Y, \varphi)$.

Démonstration. Au vu des définitions, cela résulte de $\mathcal{I}_{X} \subset \mathcal{I}_{Y}$.

Le second fait utile s'avère plus technique et s'inspire du lemme 5 de [BMZ].

Lemme 2.4. Si $\varphi \in \operatorname{End}(A)$ et si $Y$ est un sous-schéma fermé intègre de $X$ tel que $\varphi(Y)$ est un point lisse de $\varphi(X)$ alors il existe $\chi \in \operatorname{End}(V)$ tel que $\psi=\chi \circ \varphi$ satisfasse

$$
r(X, Y, \psi) \leq g-\operatorname{dim} X \quad \text { et } \quad \operatorname{rg} \psi \geq \operatorname{rg} \varphi-\operatorname{dim} \varphi(X)
$$

Démonstration. Nous notons $\eta$ le point générique de $Y$ puis $\delta^{\prime}=\delta_{\eta} \otimes \kappa(\eta)$ et $\rho=\operatorname{dim}_{\kappa(\eta)} \operatorname{Im} \delta^{\prime}$. Nous avons $\rho=g-\operatorname{dim}\left(\Omega_{X / \mathbb{C}}^{1}\right)_{\eta} \otimes \kappa(\eta) \leq g-\operatorname{dim} X$. Nous allons choisir $\psi$ avec $\operatorname{Im}^{t} \psi \subset \operatorname{Im}^{t} \varphi$ et $\left(\operatorname{Im}^{t} \psi\right)_{\eta} \otimes \kappa(\eta) \subset \operatorname{Im} \delta^{\prime}$. La première inclusion, équivalente à $\operatorname{Ker} \varphi \subset \operatorname{Ker} \psi$, montrera l'existence d'une factorisation $\psi=\chi \circ \varphi$ alors que la seconde donnera $r(X, Y, \psi)=\rho \leq g-\operatorname{dim} X$. Pour 
construire $\psi$ nous considérons le diagramme suivant à lignes exactes (voisin de celui utilisé dans la démonstration du lemme 2.1 mais en termes purement algébriques ici) où $\varphi_{1}: X \rightarrow \varphi(X)$ est le morphisme induit $\operatorname{par} \varphi$ :

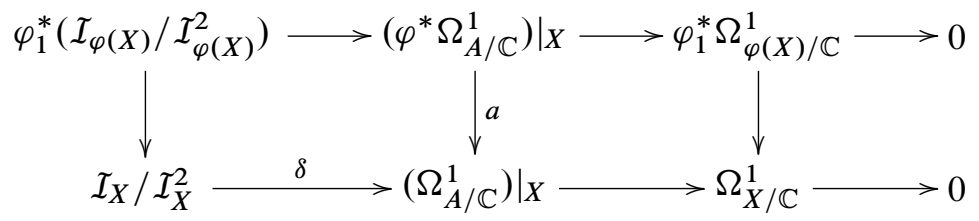

où $\operatorname{Im} a=\left.\left(\operatorname{Im}^{t} \varphi\right)\right|_{X}$. En passant aux fibres résiduelles en $\eta$, nous trouvons un second diagramme à lignes exactes

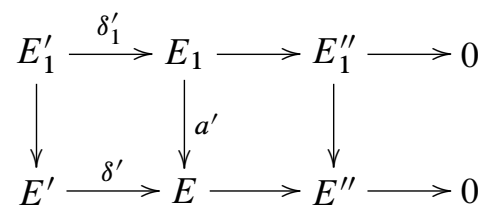

(où $E^{\prime}=\left(\mathcal{I}_{X} / \mathcal{I}_{X}^{2}\right)_{\eta} \otimes \kappa(\eta)$ et ainsi de suite). Du fait que $\varphi(\eta)$ est un point fermé, la première ligne provient d'une suite exacte sur $\kappa(\varphi(\eta))=\mathbb{C}$. En particulier, dans $E_{1}=\Gamma\left(A, \varphi^{*} \Omega_{A / \mathbb{C}}^{1}\right) \otimes_{\mathbb{C}} \kappa(\eta)$, l'image de $\delta_{1}^{\prime}$ s'écrit $F_{1} \otimes_{\mathbb{C}} \kappa(\eta)$ pour un sous- $\mathbb{C}$-espace de $\Gamma\left(A, \varphi^{*} \Omega_{A / \mathbb{C}}^{1}\right)$. Par suite, $\operatorname{Im}\left(a^{\prime} \circ \delta_{1}^{\prime}\right)=F \otimes_{\mathbb{C}} \kappa(\eta)$ pour $F \subset \Gamma\left(A, \Omega_{A / \mathbb{C}}^{1}\right)$. Il est loisible de choisir $\psi$ tel que $\operatorname{Im}^{t} \psi=F$ et nous avons alors visiblement $\operatorname{Im}^{t} \psi \subset \operatorname{Im}^{t} \varphi$ et $\left(\operatorname{Im}^{t} \psi\right)_{\eta} \otimes \kappa(\eta) \subset \operatorname{Im} \delta^{\prime}$ comme prévu. Il reste seulement à montrer la dernière inégalité de l'énoncé. D'après les définitions, $\operatorname{rg} \varphi-\operatorname{rg} \psi=\operatorname{dim} \operatorname{Im}^{t} \varphi-\operatorname{dim} \operatorname{Im}{ }^{t} \psi=\operatorname{dim} \operatorname{Im} a^{\prime}-\operatorname{dim} \operatorname{Im}\left(a^{\prime} \circ \delta_{1}^{\prime}\right) \leq$ $\operatorname{dim} E_{1}-\operatorname{dim} \operatorname{Im} \delta_{1}^{\prime}=\operatorname{dim} E_{1}^{\prime \prime}$. Maintenant cette dimension coïncide avec le rang du faisceau $\Omega_{\varphi(X) / \mathbb{C}}^{1}$ au point $\varphi(\eta)$ donc, par hypothèse de lissité, $\operatorname{dim} E_{1}^{\prime \prime}=\operatorname{dim} \varphi(X)$ et ceci donne la conclusion.

Finalement des considérations sur les nombres $r(X, Y, \varphi)$ nous permettront de borner le degré de $Y$. Pour parler de degrés, nous fixons un faisceau inversible très ample $\mathscr{L}$ sur $A$. Le résultat que nous emploierons est le suivant.

Lemme 2.5. Soient $\varphi \in \operatorname{End}(V)$ et $Y$ un sous-schéma fermé intègre de $X$. Si $r(X, Y, \varphi)<r(X, X, \varphi)$ alors il existe un fermé irréductible strict $X^{\prime}$ de $X$ contenant $Y$ tel que $\operatorname{deg} X^{\prime} \leq(\operatorname{deg} X)^{v}$ où $v=2^{g}$.

Démonstration. Il existe un entier $N$ tel que $\mathcal{I}_{X} \otimes \mathscr{L}^{\otimes N}$ est engendré par ses sections globales : par exemple, il suffit que $N$ majore la régularité de Castelnuovo-Mumford de $X$ relativement à $\mathscr{L}$ et d'après le théorème 0.2 de [Hoa] la valeur $N=(\operatorname{deg} X)^{\lambda}$ 
avec $\lambda=2^{\operatorname{dim} X}$ convient. Maintenant, grâce à la surjection $\mathcal{I}_{X} \rightarrow \mathcal{I}_{X} / \mathcal{I}_{X}^{2}$, nous constatons que $\operatorname{Im}\left(\delta_{\eta} \otimes \kappa(\eta)\right)$ est engendré par les images des sections globales de $\left(\mathcal{I}_{X} / \mathcal{I}_{X}^{2}\right) \otimes \mathscr{L}^{\otimes N}$ pour tout point $\eta$ de $X$. En rajoutant les sections de $\operatorname{Im}^{t} \varphi \otimes \mathscr{L}^{\otimes N}$, il existe une famille de sections $\left(s_{i}\right)_{i \in I}$ de $\Gamma\left(X,\left.\left(\Omega_{A / \mathbb{C}}^{1}\right)\right|_{X} \otimes \mathscr{L}^{\otimes N}\right)$ telle que $r(X, \bar{\eta}, \varphi)$ est le rang de la famille des $\left(s_{i}\right)_{\eta} \otimes \kappa(\eta) \operatorname{sur} \kappa(\eta)$ pour tout $\eta$. Quitte à permuter ces sections, nous pouvons supposer que les $\rho=r(X, X, \varphi)$ premières sont libres sur le corps des fonctions de $X$. L'hypothèse $r(X, Y, \varphi)<\rho$ impose alors qu'elles ne le sont pas $\operatorname{sur} \kappa(\eta)$ où $\bar{\eta}=Y$. En d'autres termes, la section $s=s_{1} \wedge \cdots \wedge s_{\rho}$ de $\wedge^{\rho}\left(\left.\left(\Omega_{A / \mathbb{C}}^{1}\right)\right|_{X} \otimes \mathscr{L}^{\otimes N}\right)$ est non nulle mais s'annule sur $Y$. Ce dernier faisceau s'identifie (par liberté de $\Omega_{A / \mathbb{C}}^{1}$ ) à une somme directe de copies de $\mathscr{L}^{\otimes \rho N}$ donc il existe une section de $\Gamma\left(A, \mathscr{L}^{\otimes \rho N}\right)$ s'annulant sur $Y$ mais non sur $X$. Par conséquent nous pouvons choisir pour $X^{\prime}$ une composante irréductible contenant $Y$ de l'intersection de $X$ avec le lieu des zéros de cette section. Le dit lieu est de degré $\rho N$ donc le théorème de Bézout montre $\operatorname{deg} X^{\prime} \leq \rho N(\operatorname{deg} X) \leq g(\operatorname{deg} X)^{\lambda+1}$. Pour avoir la borne de l'énoncé, il faut remarquer que $r(X, Y, \varphi)<r(X, X, \varphi)$ entraîne $0<\operatorname{dim} X<g$ (car $r(A, Y, \varphi)=\operatorname{rg} \varphi$ pour tout $Y$ ) et donc également $\operatorname{deg} X \geq 2$ (sur une variété abélienne, les seuls fermés de degré 1 sont les points). Nous concluons alors par $\log _{2} g+1+2^{g-1} \leq 2^{g}$.

Faisons ici une dernière remarque sur les degrés. Nous avons déjà observé dans la démonstration du lemme 2.2 que la somme $X+\cdots+X$ de $g$ copies de $X$ est toujours un translaté d'une sous-variété abélienne de $A$. Nous lui donnons un nom.

Définition 2.3. Nous notons $\langle X\rangle$ la plus petite sous-variété abélienne de $A$ dont un translaté contient $X$.

Nous estimons ensuite son degré.

Lemme 2.6. Pour tout sous-schéma fermé intègre $X$ de A, nous avons $\operatorname{deg}\langle X\rangle \leq$ $(\operatorname{deg} X)^{p}$ où $p=g^{2}$.

Démonstration. L'estimation est évidente si $\operatorname{dim} X=0 . \mathrm{Si} \operatorname{dim} X=1$ nous vérifions tout d'abord que $\operatorname{deg}\left(X^{\prime}+X\right) \leq 4^{\operatorname{dim} X}\left(\operatorname{deg} X^{\prime}\right)(\operatorname{deg} X)$ pour tout sous-schéma fermé intègre $X^{\prime}$ de $A$. Cette formule ne faisant pas de doute si $X^{\prime}+X=X^{\prime}$ nous pouvons supposer que $X^{\prime} \times X \rightarrow X^{\prime}+X$ est génériquement fini et ceci permet de majorer $\operatorname{deg}\left(X^{\prime}+X\right)$ par le nombre d'intersection $\left(\operatorname{add}^{*} \mathscr{L}\right) \cdot \operatorname{dim} X^{\prime}+1 \cdot X^{\prime} \times X$ (nous notons add, diff : $A \times A \rightarrow A$ les morphismes d'addition et de différence). De plus le fait que $-X$ soit numériquement équivalent à $X$ entraîne $(\operatorname{add} * \mathscr{L})^{\cdot \operatorname{dim} X^{\prime}+1}$. $X^{\prime} \times X=(\operatorname{diff} * \mathscr{L})^{\cdot \operatorname{dim}} X^{\prime}+1 \cdot X^{\prime} \times X$ et nous en déduisons $2 \operatorname{deg}\left(X^{\prime}+X\right) \leq$ $\left(\text { add }^{*} \mathscr{L} \otimes \operatorname{diff}^{*} \mathscr{L}\right)^{\cdot \operatorname{dim} X^{\prime}+1} \cdot X^{\prime} \times X$. Enfin par le théorème du cube le faisceau $\left(p_{1}^{*} \mathscr{L} \otimes p_{2}^{*} \mathscr{L}\right)^{\otimes 2} \otimes\left(\operatorname{add}^{*} \mathscr{L} \otimes \operatorname{diff}^{*} \mathscr{L}\right)^{\otimes-1}$ est numériquement effectif sur $A \times A$ 
et donc nous avons $\operatorname{deg}\left(X^{\prime}+X\right) \leq 2^{\operatorname{dim} X^{\prime}}\left(p_{1}^{*} \mathscr{L} \otimes p_{2}^{*} \mathscr{L}\right)^{\cdot \operatorname{dim} X^{\prime}+1} \cdot X^{\prime} \times X=$ $2^{\operatorname{dim} X^{\prime}}\left(\operatorname{dim} X^{\prime}+1\right)\left(\operatorname{deg} X^{\prime}\right)(\operatorname{deg} X)$ ce qui donne la formule annoncée. En raisonnant alors par récurrence, nous obtenons que le degré de la somme de $n$ copies de $X$ est au plus $2^{n^{2}-n}(\operatorname{deg} X)^{n} \leq(\operatorname{deg} X)^{n^{2}}$, d'où l'énoncé pour les courbes. Nous pouvons ramener le cas général à celui-ci de la manière suivante : $\operatorname{si} \operatorname{dim} X \geq 2$ et $\operatorname{deg}\langle X\rangle>$ $(\operatorname{deg} X)^{p}$ nous désignons par $F$ l'union (finie) des sous-variétés abéliennes $B$ de $\langle X\rangle$ avec $\operatorname{deg} B \leq(\operatorname{deg} X)^{p}$ et nous choisissons un point $x \in X$. Bien sûr nous avons $X \subset x+\langle X\rangle$ mais $X \not \subset x+F$. En coupant $X$ par le lieu des zéros de sections suffisamment générales de $\mathscr{L}$, nous pouvons trouver une courbe intègre $C \subset X$ avec $\operatorname{deg} C \leq \operatorname{deg} X, x \in C$ et $C \not \subset x+F$. Pourtant $C \subset x+\langle C\rangle$ et, par ce qui précède, $\operatorname{deg}\langle C\rangle \leq(\operatorname{deg} C)^{p} \leq(\operatorname{deg} X)^{p}$ donc $\langle C\rangle \subset F$. Cette contradiction conclut la démonstration.

Dans la suite, nous ne remplacerons pas $p$ et $v$ par leurs valeurs explicites pour ne pas alourdir les formules.

\section{Dimension des fibres}

Dans cette partie, nous montrons que le lieu des points de $X$ où la dimension des fibres d'un endomorphisme variable de $A$ est anormalement grande est un fermé. Pour pouvoir appliquer ce résultat à divers ensembles exceptionnels, nous le présentons de manière assez générale.

Si $\mu$ est une application $\operatorname{End}(V) \rightarrow \mathbb{N}$, nous posons

$$
Z_{X}(\mu)=\left\{x \in X \mid \exists \varphi \in \operatorname{End}(A) \operatorname{dim}_{x} X \cap \varphi^{-1}(\varphi(x))>\mu(\varphi)\right\} .
$$

Un théorème classique nous dit qu'à $\varphi$ fixé l'ensemble des $x$ vérifiant ceci est fermé (voir par exemple [BMZ]). Pour espérer conserver cette propriété lorsque tous les $\varphi$ entrent en jeu, il nous faut assez naturellement imposer des conditions sur $\mu$. Nous considérons l'inégalité fondamentale suivante pour $\varphi, \chi \in \operatorname{End}(V)$ :

$$
\text { (*) } 0 \leq \mu(\chi \circ \varphi)-\mu(\varphi) \leq \operatorname{rg} \varphi-\operatorname{rg} \chi \circ \varphi .
$$

Elle suffit à assurer la fermeture.

Théorème 3.1. Si (*) est vérifiée pour tout couple $(\varphi, \chi) \in \operatorname{End}(A) \times \operatorname{End}(V)$ alors $Z_{X}(\mu)$ est fermé.

La démarche de démonstration de ce théorème, basée sur les lemmes de la partie précédente, ressemble beaucoup à celle du théorème $1 \mathrm{de}$ [BMZ]. Comme dans cet article, nous montrons un résultat plus précis : dans la définition de $Z_{X}(\mu)$ il suffit de faire intervenir un nombre fini de $\varphi$. 
Pour quantifier ceci, remarquons que $\varphi$ n'intervient qu'à travers la composante neutre de son noyau car la composante de $\varphi^{-1}(\varphi(x))$ passant par $x$ est $x+\operatorname{Ker}^{0} \varphi$. En outre, si $\operatorname{Ker}^{0} \varphi=\operatorname{Ker}^{0} \varphi^{\prime}$, l'existence de deux factorisations $\varphi^{\prime}=\chi \circ \varphi$ et $\varphi=\chi^{\prime} \circ \varphi^{\prime}$ dans $\operatorname{End}(V)$ montre que $\mu(\varphi)=\mu\left(\varphi^{\prime}\right)$ sous l'hypothèse du théorème. Ceci était également le cas pour les notions $\operatorname{dim} \varphi(X)$ et $r(X, Y, \varphi)$ introduites dans la partie précédente : nous pourrions ne parler que de la variété abélienne $\operatorname{Ker}^{0} \varphi$. En particulier, si nous bornons $\operatorname{deg}\left(\operatorname{Ker}^{0} \varphi\right)$ alors nous pouvons nous contenter d'un nombre fini de $\varphi$.

De façon cohérente avec celle de [BMZ] nous posons la terminologie suivante.

Définition 3.1. Un fermé irréductible $Y$ de $A$ est dit $\mu$-anormal s'il existe $\varphi \in \operatorname{End}(A)$ tel que $\operatorname{dim} \varphi(Y)=0$ et $\operatorname{dim} Y>\mu(\varphi)$.

Il se trouve que $Z_{X}(\mu)$ est la réunion des fermés $\mu$-anormaux inclus dans $X$ : si $Y \subset X$ est $\mu$-anormal alors pour $x \in Y$ on a $Y \subset X \cap \varphi^{-1}(\varphi(x))(\operatorname{car} \varphi(Y)=\varphi(x))$ donc $x \in Z_{X}(\mu)$; réciproquement si $x \in Z_{X}(\mu)$ on choisit pour $Y$ une composante de dimension maximale passant par $x$ de $X \cap \varphi^{-1}(\varphi(x))$ et $Y$ est clairement $\mu$ anormal.

Nous montrons une borne de degré pour les fermés $\mu$-anormaux de $X$ qui sont maximaux pour cette propriété (au sens de l'inclusion).

Proposition 3.2. Sous l'hypothèse du théorème, tout fermé $\mu$-anormal maximal $Y$ de $X$ vérifie

$$
\operatorname{deg} Y \leq(\operatorname{deg} X)^{(p v+1)^{\operatorname{dim} X-1}} .
$$

Vérifions que ceci entraîne le théorème. Pour $Y$ comme dans la proposition, nous choisissons d'une part $\varphi \in \operatorname{End}(A) \operatorname{avec} \operatorname{dim} \varphi(Y)=0$ et $\operatorname{dim} Y>\mu(\varphi)$ et d'autre part $\psi \in \operatorname{End}(A)$ avec $\operatorname{Ker}^{0} \psi=\langle Y\rangle$. Comme $\operatorname{dim} \varphi(Y)=0$ entraîne que $Y$ est contenu dans un translaté de $\operatorname{Ker}^{0} \varphi$, nous avons $\operatorname{Ker}^{0} \psi \subset \operatorname{Ker}^{0} \varphi$. Ceci force $\varphi=$ $\chi \circ \psi$ pour un certain $\chi \in \operatorname{End}(V)$ donc $\mu(\varphi)=\mu(\chi \circ \psi) \geq \mu(\psi)$ d'après $(*)$. On en déduit $\operatorname{dim} \psi(Y)=0$ et $\operatorname{dim} Y>\mu(\psi)$. Enfin à l'aide de $\operatorname{deg} \operatorname{Ker}^{0} \psi \leq(\operatorname{deg} Y)^{p}$ (voir lemme 2.6) il vient

$$
\begin{array}{r}
Z_{X}(\mu)=\left\{x \in X \mid \exists \psi \in \operatorname{End}(A) \operatorname{deg} \operatorname{Ker}^{0} \psi \leq(\operatorname{deg} X)^{p(p v+1)^{\operatorname{dim} X-1}}\right. \\
\text { et } \left.\operatorname{dim}_{x} X \cap \psi^{-1}(\psi(x))>\mu(\psi)\right\}
\end{array}
$$

qui est un fermé.

Le reste de cette partie est consacré à la démonstration de la proposition. Nous supposons donc l'hypothèse du théorème vérifiée et nous procédons par récurrence sur $\operatorname{dim} X$. Les cas où $\operatorname{dim} X \leq 1$ sont triviaux car $Y$ coïncide alors avec $X(\operatorname{dim} Y=0$ est exclu par $\operatorname{dim} Y>\mu(\varphi) \in \mathbb{N}$ ) et la borne de degré vaut. 
Nous supposons désormais $\operatorname{dim} X \geq 2$ et que la proposition vaut pour tout $X^{\prime}$ avec $\operatorname{dim} X^{\prime}<\operatorname{dim} X$. Pour clarifier, nous découpons le début de l'argument en trois lemmes. Nous écrivons $\Delta=(\operatorname{deg} X)^{v(p v+1)^{\operatorname{dim} X-2}}$.

Lemme 3.1. Soit $Y$ un sous-schéma fermé intègre de $X$ pour lequel il existe $\varphi \in$ $\operatorname{End}(A)$ vérifiant $\operatorname{dim} Y>\mu(\varphi), \operatorname{dim} \varphi(Y)=0$ et $\operatorname{dim} \varphi(X)>\operatorname{dim} X-\operatorname{dim} Y$. Alors il existe un sous-schéma fermé intègre $Y^{\prime}$ de $X$ contenant $Y$ et $\mu$-anormal tel que $\operatorname{deg} Y^{\prime} \leq \Delta$.

Démonstration. D'après le lemme 2.1 appliqué à $Y$ nous avons $r(Y, Y, \varphi)=g-$ $\operatorname{dim} Y$. Grâce au lemme 2.3, il vient $r(X, Y, \varphi) \leq g-\operatorname{dim} Y$. D'un autre côté, en combinant l'hypothèse sur $\operatorname{dim} \varphi(X)$ et le lemme 2.1 appliqué maintenant à $X$, nous trouvons $r(X, X, \varphi)-g+\operatorname{dim} X>\operatorname{dim} X-\operatorname{dim} Y$. Nous en déduisons aisément $r(X, Y, \varphi)<r(X, X, \varphi)$ de sorte que le lemme 2.5 fournit $X^{\prime}$ avec $\operatorname{dim} X^{\prime}<\operatorname{dim} X$, $Y \subset X^{\prime} \subset X$ et $\operatorname{deg} X^{\prime} \leq(\operatorname{deg} X)^{v}$. Ensuite, le fermé $Y$, étant $\mu$-anormal, est contenu dans un fermé $\mu$-anormal maximal $Y^{\prime}$ de $X^{\prime}$. Par hypothèse de récurrence $\operatorname{deg} Y^{\prime} \leq\left(\operatorname{deg} X^{\prime}\right)^{(p v+1)^{\operatorname{dim} X^{\prime}-1}} \leq \Delta$.

Nous remplaçons maintenant la condition $\operatorname{dim} \varphi(X)>\operatorname{dim} X-\operatorname{dim} Y$ par une hypothèse de lissité.

Lemme 3.2. Soit $Y$ un sous-schéma fermé intègre de $X$ pour lequel il existe $\varphi \in$ $\operatorname{End}(A)$ tel que $\operatorname{dim} Y>\mu(\varphi)$ et $\varphi(Y)$ est un point lisse de $\varphi(X)$. Alors il existe un sous-schéma fermé intègre $Y^{\prime}$ de $X$ contenant $Y$ et $\mu$-anormal tel que $\operatorname{deg} Y^{\prime} \leq \Delta$.

Démonstration. Nous pouvons supposer $\operatorname{dim} \varphi(X) \leq \operatorname{dim} X-\operatorname{dim} Y$ car sinon le lemme précédent suffit. Nous appliquons alors le lemme 2.4 qui nous donne $\psi=\chi \circ \varphi \in \operatorname{End}(V)$ avec $r(X, Y, \psi) \leq g-\operatorname{dim} X$ et $\operatorname{rg} \psi \geq \operatorname{rg} \varphi-\operatorname{dim} \varphi(X)$. Si jamais $r(X, Y, \psi)<r(X, X, \psi)$ alors nous concluons exactement comme dans la démonstration précédente avec le lemme 2.5. Dans le cas contraire, nous avons $r(X, X, \psi) \leq g-\operatorname{dim} X$ d'où (lemme 2.1) $\operatorname{dim} \psi(X)=0$. Ici le lemme 2.2 entre en jeu et montre que nous pouvons écrire $\psi=\chi^{\prime} \circ \varphi^{\prime}$ avec $\varphi^{\prime} \in \operatorname{End}(A)$ et $\operatorname{dim} \varphi^{\prime}(X)=0$. Nous tirons de $(*)$

$$
\operatorname{rg} \varphi+\mu(\varphi) \geq \operatorname{rg} \psi+\mu(\psi) \quad \text { et } \quad \mu(\psi) \geq \mu\left(\varphi^{\prime}\right) .
$$

En combinant ceci et les inégalités

$$
\operatorname{dim} Y>\mu(\varphi), \quad \operatorname{rg} \psi \geq \operatorname{rg} \varphi-\operatorname{dim} \varphi(X) \quad \text { et } \quad \operatorname{dim} \varphi(X) \leq \operatorname{dim} X-\operatorname{dim} Y,
$$

nous voyons apparaitre $\operatorname{dim} X>\mu\left(\varphi^{\prime}\right)$. Ainsi $X$ est $\mu$-anormal donc $Y^{\prime}=X$ convient. 
Notre troisième étape est, elle, indépendante de la partie précédente.

Lemme 3.3. Soient $\varphi, \psi \in \operatorname{End}(A)$ et $Y, Z$ deux sous-schémas fermés intègres de $X$ de sorte que $\operatorname{dim} Y=\operatorname{dim} Z$ et $\operatorname{dim} \varphi(Y)=\operatorname{dim} \varphi(Z)=\operatorname{dim} \psi(Z)=0$. On suppose en outre $\operatorname{dim} \varphi(X)=\operatorname{dim} X-\operatorname{dim} Y$ et que $Y$ est une composante irréductible de $X \cap \varphi^{-1}(\varphi(Y))$. Alors $\operatorname{dim} \psi(Y)=0$.

Démonstration. Nous considérons $(\varphi, \psi): A \rightarrow A \times A$ et sa restriction $f$ à $X$. Comme $f(Z)$ est un point, la dimension relative de $f$ est au moins $\operatorname{dim} Z=\operatorname{dim} Y$. Autrement dit nous avons $\operatorname{dim} f(X) \leq \operatorname{dim} X-\operatorname{dim} Y=\operatorname{dim} \varphi(X)$. Comme il y a un morphisme surjectif évident $f(X) \rightarrow \varphi(X)$, ces deux schémas ont en fait même dimension et donc la dimension relative de $f$ est $\operatorname{dim} Y$. Écrivons maintenant $X \cap \varphi^{-1}(\varphi(Y))=Y \cup S$ où $S$ est un fermé ne contenant pas $Y$ (l'union des autres composantes irréductibles par exemple) et choisissons $y \in Y$ avec $y \notin S$. Considérons enfin une composante irréductible $\widetilde{Y}$ de $f^{-1}(f(y))$ contenant $y$. Puisque $f(\tilde{Y})=f(y)$ nous avons a fortiori $\varphi(\tilde{Y})=\varphi(y)=\varphi(Y)$ donc $\tilde{Y} \subset Y \cup S$. L'inclusion $\tilde{Y} \subset S$ est interdite par $y \in \tilde{Y}$ donc $\tilde{Y} \subset Y$. Par ailleurs, en tant que composante irréductible d'une fibre de $f$, le fermé $\tilde{Y}$ est de dimension au moins égale à la dimension relative de ce morphisme. Ceci signifie $\operatorname{dim} \tilde{Y} \geq \operatorname{dim} Y$ et donc $\widetilde{Y}=Y$. Nous en déduisons $f(Y)=f(y)$ puis $\psi(Y)=\psi(y)$ par projection.

Nous terminons maintenant la démonstration de la proposition. Soit donc $Y$ un fermé $\mu$-anormal maximal de $X$ et fixons $\varphi \in \operatorname{End}(A) \operatorname{avec} \operatorname{dim} Y>\mu(\varphi)$ et $\operatorname{dim} \varphi(Y)=0$. Si nous avons $\operatorname{dim} \varphi(X)>\operatorname{dim} X-\operatorname{dim} Y$ alors le lemme 3.1 nous permet de conclure immédiatement : il vient en effet $Y=Y^{\prime}$ par maximalité et donc $\operatorname{deg} Y \leq \Delta$ ce qui est inférieur à la borne de la proposition.

Supposons donc $\operatorname{dim} \varphi(X) \leq \operatorname{dim} X-\operatorname{dim} Y$. Ceci signifie que la dimension relative de $\left.\varphi\right|_{X}$ est au moins $\operatorname{dim} Y$ mais nous avons nécessairement égalité car $Y$ est composante d'une fibre de $\left.\varphi\right|_{X}$ par maximalité : toute composante de $X \cap \varphi^{-1}(\varphi(Y))$ de dimension au moins $\operatorname{dim} Y>\mu(\varphi)$ est automatiquement $\mu$-anormale. Nous pouvons donc trouver un ouvert $U$ non vide de $\varphi(X)$ tel que toutes les fibres des points de $U$ soient équidimensionnelles de dimension $\operatorname{dim} Y$. Quitte à restreindre cet ouvert, nous le supposons contenu dans le lieu lisse de $\varphi(X)$. De cette façon, si $u \in U$ et si $Z$ est une composante irréductible de $X \cap \varphi^{-1}(u)$, le lemme 3.2 s'applique à $Z$ (car $\operatorname{dim} Z=\operatorname{dim} Y>\mu(\varphi)$ et $\varphi(Z)=u$ est lisse). Tout tel $Z$ est donc contenu dans un $Z^{\prime} \mu$-anormal avec $\operatorname{deg} Z^{\prime} \leq \Delta$. En vertu de la majoration $\operatorname{deg}\left\langle Z^{\prime}\right\rangle \leq \Delta^{p}$, la variété abélienne $\left\langle Z^{\prime}\right\rangle$ appartient à un ensemble fini (indépendant de $Z$ ). Nous pouvons donc choisir $\psi_{1}, \ldots, \psi_{n} \in \operatorname{End}(A)$ de sorte que $\left\langle Z^{\prime}\right\rangle$ soit l'un des $\operatorname{Ker}^{0} \psi_{i}$ (et nous imposons que tout $\operatorname{Ker}^{0} \psi_{i}$ apparaisse effectivement de cette façon). Par l'argument donné immédiatement après l'énoncé de la propostion, nous avons $\operatorname{dim} Z^{\prime}>\mu\left(\psi_{i}\right)$ comme conséquence de $(*)$. 
Appliquons enfin notre lemme 3.3 à $Y, Z, \varphi, \psi_{i}$. Nous avons bien $\operatorname{dim} \psi_{i}(Z)=0$ car $Z \subset Z^{\prime}$ est contenu dans un translaté de $\left\langle Z^{\prime}\right\rangle=\operatorname{Ker}^{0} \psi_{i}$ et $Y$ est une composante de $X \cap \varphi^{-1}(\varphi(Y))$. De cette façon, il vient $\operatorname{dim} \psi_{i}(Y)=0$.

Notre dernière tâche consiste à montrer que $Y$ est composante de $X \cap \psi_{i}^{-1}\left(\psi_{i}(Y)\right)$ pour au moins un indice $i$. Si ce n'était pas le cas, nous aurions $Y \subset Y_{i} \subset X \cap$ $\psi_{i}^{-1}\left(\psi_{i}(Y)\right)$ pour une certaine composante $Y_{i}$ avec $\operatorname{dim} Y_{i}>\operatorname{dim} Y$ pour tout $i$. Pour que ce fermé ne contredise pas la maximalité de $Y$, il doit nécessairement vérifier $\operatorname{dim} Y_{i} \leq \mu\left(\psi_{i}\right)$. Par suite le lieu

$$
F_{i}=\left\{x \in X \mid \operatorname{dim}_{x} X \cap \psi_{i}^{-1}\left(\psi_{i}(x)\right)>\mu\left(\psi_{i}\right)\right\}
$$

est un fermé strict de $X$. Il en va de même de l'union finie $F=F_{1} \cup \cdots \cup F_{n}$. Par ailleurs, nous avons vu $\operatorname{dim} Z^{\prime}>\mu\left(\psi_{i}\right)$ qui donne $Z \subset Z^{\prime} \subset F$. Comme $Z$ peut être n'importe quelle composante d'une fibre au-dessus de $U$, nous aurions $X \cap \varphi^{-1}(U) \subset F$, ce qui contredit clairement la densité de $U$.

Notre $Y$ est donc finalement composante d'une intersection de la forme $X \cap$ $y+\operatorname{Ker}^{0} \psi_{i}$ donc le théorème de Bézout montre $\operatorname{deg} Y \leq(\operatorname{deg} X)\left(\operatorname{deg} \operatorname{Ker}^{0} \psi_{i}\right) \leq$ $(\operatorname{deg} X) \Delta^{p}$ et ceci conclut la démonstration de la propostion en vertu de l'inégalité $1+p v(p v+1)^{\operatorname{dim} X-2} \leq(p v+1)^{\operatorname{dim} X-1}$.

Comme nous l'avons dit, cette preuve se rapproche beaucoup des arguments de [BMZ] (la démonstration du lemme 3.3 en particulier est presque identique à un passage de ce texte). Toutefois, à la différence de cet article, nous procédons en un seul temps en raisonnant sur le degré de $Y$ plutôt que sur le degré d'un $\varphi$ tel que $\operatorname{dim} \varphi(Y)=0$.

\section{Ensembles exceptionnels}

Dans cette partie, nous établissons le théorème 1.4 en montrant que chacun des ensembles qui nous intéressent s'écrit sous la forme $Z_{X}(\mu)$ étudiée dans la partie précédente et donc est fermé. Nous obtiendrons aussi de cette façon les différentes inclusions de l'énoncé en utilisant le fait évident sur la définition que si $\mu \geq \mu^{\prime}$ alors $Z_{X}(\mu) \subset Z_{X}\left(\mu^{\prime}\right)$.

Notre tâche consiste donc à définir trois fonctions $\mu$. Ici encore, elles s'inscrivent dans un cadre commun. Nous considérons un entier $r$ avec $0 \leq r \leq g$ et un sousanneau $\mathcal{O} \subset \operatorname{End}(V)$ auxquels nous associons la fonction $\mu_{\mathcal{O}, r}: \operatorname{End}(V) \rightarrow \mathbb{N}$ définie par

$$
\mu_{\mathcal{O}, r}(\varphi)= \begin{cases}0 & \text { si } \operatorname{rg} \varphi \geq r, \\ \inf _{\substack{\left(\psi, \varphi^{\prime}\right) \in \operatorname{End}(V) \times \mathcal{O} \\ \varphi=\psi \circ \varphi^{\prime} \text { et } \operatorname{rg} \varphi^{\prime} \geq r}} \operatorname{rg} \varphi^{\prime}-\operatorname{rg} \varphi-1 & \text { sinon. }\end{cases}
$$


On notera que la définition a bien un sens car dans le second cas on a $\operatorname{rg} \varphi^{\prime} \geq r>$ $\operatorname{rg} \varphi$ donc $\operatorname{rg} \varphi^{\prime}-\operatorname{rg} \varphi-1 \geq 0$ : on prend bien l'infimum d'une partie de $\mathbb{N}$ et elle n'est pas vide car le couple $\left(\psi, \varphi^{\prime}\right)=\left(\varphi, \mathrm{id}_{V}\right)$ convient toujours en vertu de $g \geq r$. Pour deux sous-anneaux $\mathcal{O}$ et $\mathcal{O}^{\prime}$ nous avons immédiatement

$$
\mathcal{O} \subset \mathcal{O}^{\prime} \Longrightarrow \mu_{\mathcal{O}, r} \geq \mu_{\mathcal{O}^{\prime}, r} \Rightarrow Z_{X}\left(\mu_{\mathcal{O}, r}\right) \subset Z_{X}\left(\mu_{\mathcal{O}^{\prime}, r}\right)
$$

Il nous reste à examiner la condition $(*)$ apparaissant dans la partie précédente. Nous l'obtenons moyennant une hypothèse supplémentaire sur $\mathcal{O}$.

Lemme 4.1. Si tout idéal à gauche de $\mathcal{O}$ est monogène alors la fonction $\mu_{\mathcal{O}, r}$ satisfait (*) pour tous $\varphi \in \mathcal{O}$ et $\chi \in \operatorname{End}(V)$.

Démonstration. Nous notons ici simplement $\mu=\mu_{\mathcal{O}, r}$. Nous vérifions d'abord $\mu(\chi \circ \varphi)-\mu(\varphi) \leq \operatorname{rg} \varphi-\operatorname{rg} \chi \circ \varphi$. Si $\mu(\chi \circ \varphi)=0$, ceci est clair $\operatorname{car} \operatorname{rg} \chi \circ \varphi \leq \operatorname{rg} \varphi$. Si $\mu(\chi \circ \varphi)>0$ et $\operatorname{rg}(\varphi) \geq r$, nous choisissons simplement $\psi=\chi$ et $\varphi^{\prime}=\varphi \in \mathcal{O}$ dans l'infimum définissant $\mu(\chi \circ \varphi)$ et trouvons $\mu(\chi \circ \varphi) \leq \operatorname{rg} \varphi-\operatorname{rg} \chi \circ \varphi-1$ qui donne le résultat. Si maintenant $\operatorname{rg} \chi \circ \varphi \leq \operatorname{rg} \varphi<r$, nous choisissons un couple $\left(\psi, \varphi^{\prime}\right)$ réalisant l'infimum dans la définition de $\mu(\varphi)$ de sorte que $\mu(\varphi)=\operatorname{rg} \varphi^{\prime}-\operatorname{rg} \varphi-1$; le simple fait d'écrire $\chi \circ \varphi=(\chi \circ \psi) \circ \varphi^{\prime}$ montre $\mu(\chi \circ \varphi) \leq \operatorname{rg} \varphi^{\prime}-\operatorname{rg} \chi \circ \varphi-1=$ $\operatorname{rg} \varphi-\operatorname{rg} \chi \circ \varphi-\mu(\varphi):$ c'est notre résultat.

Dans un deuxième temps, nous montrons $\mu(\varphi) \leq \mu(\chi \circ \varphi)$. Là encore, il n'y a rien à faire si $\mu(\varphi)=0$ donc nous pouvons supposer $\operatorname{rg} \chi \circ \varphi \leq \operatorname{rg} \varphi<r$. Nous choisissons cette fois-ci un couple $\left(\psi, \varphi^{\prime}\right)$ réalisant l'infimum pour $\mu(\chi \circ \varphi)$ : nous avons $\chi \circ \varphi=$ $\psi \circ \varphi^{\prime}, \varphi^{\prime} \in \mathcal{O}, \operatorname{rg} \varphi^{\prime} \geq r$ et $\mu(\chi \circ \varphi)=\operatorname{rg} \varphi^{\prime}-\operatorname{rg} \chi \circ \varphi-1$. Par hypothèse, l'idéal $\mathcal{O} \varphi+\mathcal{O} \varphi^{\prime}$ de $\mathcal{O}$ est monogène ; nous l'écrivons $\mathcal{O} \varphi^{\prime \prime}$ avec $\varphi^{\prime \prime} \in \mathcal{O}$. En particulier, nous pouvons écrire $\varphi^{\prime}=\psi^{\prime \prime} \circ \varphi^{\prime \prime}$ qui montre $\operatorname{rg} \varphi^{\prime \prime} \geq \operatorname{rg} \varphi^{\prime} \geq r$ et $\varphi=\psi^{\prime} \circ \varphi^{\prime \prime}$ qui montre $\mu(\varphi) \leq \operatorname{rg} \varphi^{\prime \prime}-\operatorname{rg} \varphi-1$ par définition de $\mu(\varphi)$. En comparant avec la valeur de $\mu(\chi \circ \varphi)$, il nous suffira de montrer $\operatorname{rg} \varphi^{\prime \prime} \leq \operatorname{rg} \varphi+\operatorname{rg} \varphi^{\prime}-\operatorname{rg} \chi \circ \varphi$ pour conclure. Or l'égalité $\mathcal{O} \varphi+\mathcal{O} \varphi^{\prime}=\mathcal{O} \varphi^{\prime \prime}$ nous donne encore une relation $\varphi^{\prime \prime}=\chi_{1} \circ \varphi+\chi_{2} \circ \varphi^{\prime}$ qui entraîne $\operatorname{Ker} \varphi \cap \operatorname{Ker} \varphi^{\prime} \subset \operatorname{Ker} \varphi^{\prime \prime}$. Comme par ailleurs $\chi \circ \varphi=\psi \circ \varphi^{\prime}$ implique facilement $\operatorname{Ker} \varphi+\operatorname{Ker} \varphi^{\prime} \subset \operatorname{Ker} \chi \circ \varphi$, nous trouvons $\operatorname{rg} \varphi^{\prime \prime}+\operatorname{rg} \chi \circ \varphi=2 g-\operatorname{dim} \operatorname{Ker} \varphi^{\prime \prime}-$ $\operatorname{dim} \operatorname{Ker} \chi \circ \varphi \leq 2 g-\operatorname{dim}\left(\operatorname{Ker} \varphi \cap \operatorname{Ker} \varphi^{\prime}\right)-\operatorname{dim}\left(\operatorname{Ker} \varphi+\operatorname{Ker} \varphi^{\prime}\right)=\operatorname{rg} \varphi+\operatorname{rg} \varphi^{\prime}$ et le lemme est démontré.

Disons tout de suite à quels anneaux nous appliquerons ceci.

Lemme 4.2. Dans chacun des trois anneaux $\operatorname{End}(A) \otimes \mathbb{Q} \subset \operatorname{End}(A) \otimes \mathbb{R} \subset \operatorname{End}(V)$, tout idéal à gauche est monogène.

Démonstration. Chacun est produit d'anneaux de matrices sur des corps (non nécessairement commutatifs pour les deux premiers) : voir par exemple [Mu, p. 174]. 
Comme $\operatorname{End}(A)$ est contenu dans chacun des trois anneaux ci-dessus, nous avons bien, par le théorème 3.1 et ces deux lemmes, trois fermés emboîtés

$$
Z_{X}\left(\mu_{\operatorname{End}(A) \otimes \mathbb{Q}, r}\right) \subset Z_{X}\left(\mu_{\operatorname{End}(A) \otimes \mathbb{R}, r}\right) \subset Z_{X}\left(\mu_{\operatorname{End}(V), r}\right)
$$

et il ne reste plus qu'à les identifier aux ensembles exceptionnels du théorème 1.4. C'est immédiat pour le dernier, facile pour le premier mais plus délicat pour le second. Nous procédons par ordre croissant de difficulté.

Lemme 4.3. Nous avons $Z_{X}\left(\mu_{\operatorname{End}(V), r}\right)=Z_{X \text {,an }}^{(r)}$.

Démonstration. Si l'on se reporte à la définition rappelée dans l'introduction, l'on constate que $Z_{X \text {,an }}^{(r)}$ est déjà écrit sous la forme $Z_{X}(\mu)$ où $\mu$ est la fonction donnée $\operatorname{par} \mu(\varphi)=\max (0, r-1-\operatorname{rg} \varphi)$. Vérifions simplement $\mu_{\operatorname{End}(V), r}=\mu$. Si $\operatorname{rg} \varphi \geq r$, l'égalité est claire. Sinon il suffit de voir que l'on peut choisir dans l'infimum $\operatorname{rg} \varphi^{\prime}=r$ : on se contente de fixer un sous-espace $E$ de $\operatorname{Ker} \varphi$ de dimension $g-r$ et l'on impose $\operatorname{Ker} \varphi^{\prime}=E$ de façon à ce que l'inclusion $\operatorname{Ker} \varphi^{\prime} \subset \operatorname{Ker} \varphi$ donne la factorisation $\varphi=\psi \circ \varphi^{\prime}$.

Nous en venons à $Z_{X, \mathbb{Q}}^{(r)}$. Par définition ([R2, p. 319]) il s'agit de l'union de tous les fermés irréductibles de $X$ qui ne sont pas $r$-QQ-supertransverses. En outre un fermé irréductible $Y$ est $r$ - $\mathbb{Q}$-supertransverse si et seulement si ([R2, lemme 7.2] et la remarque qui le suit) pour toute sous-variété abélienne $B$ de $A$ de codimension au moins $r$ et toute sous-variété abélienne $B^{\prime}$ de $A$ contenant strictement $B$ on a $\operatorname{dim} Y+B^{\prime}>\operatorname{dim} Y+B=\operatorname{dim} Y+\operatorname{dim} B$.

Lemme 4.4. Nous avons $\mu_{\operatorname{End}(A), r}=\mu_{\operatorname{End}(A) \otimes \mathbb{Q}, r}$ et $Z_{X}\left(\mu_{\operatorname{End}(A), r}\right)=Z_{X, \mathbb{Q}}^{(r)}$.

Démonstration. L'égalité des fonctions $\mu$ résulte du fait que si l'on peut écrire $\varphi=$ $\psi \circ \varphi^{\prime}$ avec $\varphi^{\prime} \in \operatorname{End}(A) \otimes \mathbb{Q}$ alors $\varphi=\left(\frac{1}{N} \psi\right) \circ\left(N \varphi^{\prime}\right)$ pour un certain entier $N \in \mathbb{N} \backslash\{0\}$ tel que $N \varphi^{\prime} \in \operatorname{End}(A)$.

Si $\varphi \in \operatorname{End}(A)$ et $B=\operatorname{Ker}^{0} \varphi$ alors pour tout fermé $Y$ irréductible nous avons $\operatorname{dim} Y+B=\operatorname{dim} \varphi(Y)+\operatorname{dim} B$ et $\operatorname{dim} B=g-\operatorname{rg} \varphi$. Par conséquent la condition que $Y$ est $r-\mathbb{Q}$-supertransverse peut se traduire par les deux conditions suivantes (faire $B=\operatorname{Ker}^{0} \varphi$ et $\left.B^{\prime}=\operatorname{Ker}^{0} \psi\right)$.

(a) Pour tout $\varphi \in \operatorname{End}(A) \operatorname{avec} \operatorname{rg} \varphi \geq r$ on $\operatorname{adim} \varphi(Y)=\operatorname{dim} Y$.

(b) Pour tout $\psi \in \operatorname{End}(A)$ tel qu'il existe $\varphi \in \operatorname{End}(A), \chi \in \operatorname{End}(V)$ avec $\psi=\chi \circ \varphi$, $\operatorname{rg} \varphi \geq r$ et $\operatorname{rg} \varphi>\operatorname{rg} \psi$, on $\operatorname{dim} \psi(Y)>\operatorname{dim} Y+\operatorname{rg} \psi-\operatorname{rg} \varphi$.

Nous remarquons que, si (a) est vérifiée, alors (b) est automatiquement vraie pour les $\psi$ tels que $\operatorname{rg} \psi \geq r$. Nous pouvons donc limiter (b) aux $\psi$ avec $\operatorname{rg} \psi<$ $r$, ce qui permet de supprimer la condition $\operatorname{rg} \varphi>\operatorname{rg} \psi$. En outre puisque l'on a toujours $\operatorname{dim} \varphi(Y) \leq \operatorname{dim} Y$, nous pouvons écrire deux conditions équivalentes à la conjonction de (a) et (b) sous la forme suivante. 
(a') Pour tout $\varphi \in \operatorname{End}(A)$ avec $\operatorname{rg} \varphi \geq r$ on $\operatorname{adim} \varphi(Y) \geq \operatorname{dim} Y$.

$\left(\mathrm{b}^{\prime}\right)$ Pour tout $\psi \in \operatorname{End}(A)$ avec $\operatorname{rg} \psi<r$ on a

$$
\operatorname{dim} \psi(Y)>\operatorname{dim} Y+\sup _{\substack{(\chi, \varphi) \in \operatorname{End}(V) \times \operatorname{End}(A) \\ \psi=\chi \circ \varphi \text { et } \operatorname{rg} \varphi \geq r}} \operatorname{rg} \psi-\operatorname{rg} \varphi .
$$

Nous voyons ainsi apparaître la fonction $\mu=\mu_{\operatorname{End}(A), r}$. En effet ces deux conditions se réduisent purement et simplement à : $\forall \varphi \in \operatorname{End}(A), \operatorname{dim} \varphi(Y) \geq \operatorname{dim} Y-$ $\mu(\varphi)$. Par suite un point $x$ appartient à $Z_{X, \mathbb{Q}}^{(r)}$ si et seulement s'il existe $\varphi \in \operatorname{End}(A)$ et un fermé irréductible $Y$ de $X$ contenant $x$ tel que $\operatorname{dim} \varphi(Y)<\operatorname{dim} Y-\mu(\varphi)$.

Nous en déduisons l'inclusion $Z_{X}(\mu) \subset Z_{X, \mathbb{Q}}^{(r)}$ car un fermé $\mu$-anormal vérifie clairement cette condition. En sens contraire, l'inégalité $\operatorname{dim} \varphi(Y)<\operatorname{dim} Y-\mu(\varphi)$ signifie que la dimension relative $d$ du morphisme restreint $Y \rightarrow \varphi(Y)$ vérifie $d>$ $\mu(\varphi)$. Comme toutes les composantes des fibres de ce morphisme sont de dimension au moins $d$, elles sont $\mu$-anormales donc $Y \subset Z_{X}(\mu)$ puis par union $Z_{X, \mathbb{Q}}^{(r)} \subset$ $Z_{X}(\mu)$.

Il nous reste à traiter de $Z_{X}^{(r)}$. Nous commençons par revenir sur la définition rappelée dans l'introduction (voir aussi [R2, p. 319]). Celle-ci fait intervenir le cône $Z_{+}^{1}(A)$ des diviseurs effectifs à coefficients réels sur $A$ modulo équivalence numérique. Ici nous préférons mettre l'accent sur les endomorphismes aussi utiliserons-nous le fait ([R2, lemme 7.1]) que, pour un faisceau inversible ample $\mathscr{L}$ sur $A$ désormais fixé,

$$
Z_{+}^{1}(A)=\left\{\varphi^{*} \mathscr{L} \mid \varphi \in \operatorname{End}(A) \otimes \mathbb{R}\right\}
$$

(l'application quadratique $\operatorname{End}(A) \rightarrow \mathrm{NS}(A), \mathscr{L} \mapsto \varphi^{*} \mathscr{L}$ s'étend en $\operatorname{End}(A) \otimes \mathbb{R} \rightarrow$ $\mathrm{NS}(A) \otimes \mathbb{R})$. Avec cette reformulation, la définition s'exprime comme suit :

(1) $Z_{X}^{(r)}$ est l'union de tous les fermés irréductibles de $X$ qui ne sont pas $r$-supertransverses ;

(2) un fermé irréductible $Y$ de $X$ est dit $r$-supertransverse si pour tous $\varphi, \varphi_{1}, \varphi_{2}$ dans $\operatorname{End}(A) \otimes \mathbb{R}$ et $a \in \mathbb{N}$ vérifiant $\operatorname{rg} \varphi \geq r, \varphi^{*} \mathscr{L}=\varphi_{1}^{*} \mathscr{L}+\varphi_{2}^{*} \mathscr{L}$ et $\operatorname{rg} \varphi_{1}-1 \leq$ $a \leq \operatorname{dim} Y$ on a $\left(\varphi^{*} \mathscr{L}\right)^{\cdot a} \cdot\left(\varphi_{2}^{*} \mathscr{L}\right)^{\cdot \operatorname{dim} Y-a} \cdot Y>0$.

Notre première tâche consiste à reformuler la condition sur le nombre d'intersections en termes des entiers $\operatorname{dim} \varphi(Y)$ et $\operatorname{dim} \varphi_{2}(Y)$ définis dans la partie 2 (en vue de se rapprocher des assertions (a) et (b) apparues dans la démonstration précédente). Nous transitons par une intégrale.

Nous notons $H: V \times V \rightarrow \mathbb{C}$ la forme de Riemann de $\mathscr{L}$ et écrivons $H_{\varphi}$ pour la forme hermitienne $\left(v, v^{\prime}\right) \mapsto H\left(\varphi(v), \varphi\left(v^{\prime}\right)\right)$ lorsque $\varphi \in \operatorname{End}(V)$. Nous désignons par $\omega=-\operatorname{Im} H$ l'opposé de la partie imaginaire de $H$ que nous voyons comme une $(1,1)$-forme de Kähler sur $V$ ou sur $A$. De même nous écrivons $\omega_{\varphi}=-\operatorname{Im} H_{\varphi}$. 
Dans les trois lemmes suivants un sous-schéma fermé intègre $Y$ de $A$ est temporairement fixé. Nous rappelons que $\pi$ désigne la projection $V \rightarrow V / \Lambda=A$. Nous désignons par $F$ un domaine fondamental dans $V$ pour ce quotient et écrivons $\hat{Y}=\pi^{-1}(Y) \cap F$.

Lemme 4.5. Pour tous $\varphi, \psi \in \operatorname{End}(A) \otimes \mathbb{R}$ et tout $a \in \mathbb{N}$ on $a$

$$
\left(\varphi^{*} \mathscr{L}\right)^{\cdot a} \cdot\left(\psi^{*} \mathscr{L}\right)^{\cdot \operatorname{dim} Y-a} \cdot Y=\int_{\hat{Y}} \omega_{\varphi}^{\wedge a} \wedge \omega_{\psi}^{\wedge \operatorname{dim} Y-a}
$$

Démonstration. Pour tout faisceau $\mathcal{N} \in \mathrm{NS}(A)$ de forme de Riemann $H_{\mathcal{N}}$ nous avons $\mathcal{N}^{\cdot \operatorname{dim} Y} \cdot Y=\int_{Y} \omega_{\mathcal{N}}^{\wedge \operatorname{dim} Y}=\int_{\hat{Y}} \omega_{\mathcal{N}}^{\wedge \operatorname{dim} Y}$ car la forme $\omega_{\mathcal{N}}=-\operatorname{Im} H_{\mathcal{N}}$ correspond à la première classe de Chern de $\mathcal{N}$ (voir [Mu, p. 18]). En écrivant $\mathcal{N}=$ $a_{1} \mathcal{N}_{1}+\cdots+a_{\operatorname{dim} Y} \mathcal{N}_{\operatorname{dim} Y}$ dans $\mathrm{NS}(A)$ où $a_{i} \in \mathbb{Z}$ et en développant les deux membres, polynômes homogènes de degré $\operatorname{dim} Y$ en les $a_{i}$, puis en identifiant les coefficients du produit $a_{1} \cdots a_{\operatorname{dim} Y}$ il vient

$$
\mathcal{N}_{1} \ldots \mathcal{N}_{\operatorname{dim} Y} \cdot Y=\int_{\hat{Y}} \omega_{\mathcal{N}_{1}} \wedge \cdots \wedge \omega_{\mathcal{N}_{\operatorname{dim} Y}} .
$$

En choisissant $\mathcal{N}_{i}=\varphi^{*} \mathscr{L}$ si $i \leq a$ et $\mathcal{N}_{i}=\psi^{*} \mathscr{L}$ sinon nous obtenons la formule de l'énoncé si $\varphi, \psi \in \operatorname{End}(A)$ car la forme de Riemann de $\varphi^{*} \mathscr{L}$ coïncide avec $H_{\varphi}$. Ensuite cette identité polynomiale sur $\operatorname{End}(A)$ s'étend à nouveau à $\operatorname{End}(A) \otimes \mathbb{R}$.

Voici la traduction en termes de dimension.

Lemme 4.6. Soient $\varphi, \psi \in \operatorname{End}(A) \otimes \mathbb{R}$. L'inégalité $\left(\varphi^{*} \mathscr{L}\right)^{\cdot \operatorname{dim} Y} \cdot Y>0$ est équivalente à $\operatorname{dim} \varphi(Y)=\operatorname{dim} Y$ et, si elle est vérifiée et $a \in \mathbb{N}$ avec $a \leq \operatorname{dim} Y$ est donné, l'inégalité $\left(\varphi^{*} \mathscr{L}\right)^{\cdot a} \cdot\left(\psi^{*} \mathscr{L}\right)^{\cdot \operatorname{dim} Y-a} \cdot Y>0$ équivaut à $\operatorname{dim} \psi(Y) \geq \operatorname{dim} Y-a$.

Démonstration. Nous recouvrons un ouvert dense de $\hat{Y}$ par des ouverts lisses $U$ tels que $\varphi(U)$ est une variété lisse de dimension $\operatorname{dim} \varphi(Y)$. Le nombre $\left(\varphi^{*} \mathscr{L}\right)^{\cdot \operatorname{dim} Y} \cdot Y$ est somme des $\int_{U} \omega_{\varphi}^{\wedge \operatorname{dim} Y}$ et, si $\varphi^{\prime}: U \rightarrow \varphi(U)$ est induit par $\varphi$, alors $\omega_{\varphi}^{\operatorname{dim} Y}=$ $\left(\varphi^{\prime}\right)^{*}\left(\omega^{\wedge \operatorname{dim} Y}\right) . \operatorname{Si} \operatorname{dim} \varphi(U)=\operatorname{dim} \varphi(Y)<\operatorname{dim} Y$ alors cette forme est nulle; sinon c'est une forme volume sur $U$ et donc $\int_{U} \omega_{\varphi}^{\wedge \operatorname{dim} Y}>0$. Ceci montre la première équivalence.

Pour la seconde, nous pouvons supposer que $\psi(U)$ est également lisse de dimension $\operatorname{dim} \psi(Y)$ et montrer seulement $\int_{U} \omega_{\varphi}^{\wedge a} \wedge \omega_{\psi}^{\wedge \operatorname{dim} Y-a}>0 \Longleftrightarrow \operatorname{dim} \psi(Y) \geq$ $\operatorname{dim} Y-a$. Là encore, si $\operatorname{dim} \psi(Y)<\operatorname{dim} Y-a$, la forme $\omega_{\psi}^{\wedge \operatorname{dim} Y-a}$ provient d'une variété de dimension strictement inférieure à son degré donc est nulle sur $U$. Si $\operatorname{dim} \psi(Y) \geq \operatorname{dim} Y-a$, elle est au contraire non nulle $:\left.\left(\omega_{\psi}^{\operatorname{dim} Y-a}\right)\right|_{U} \neq 0$. Comme de son côté $\left.\left(\omega_{\varphi}\right)\right|_{U}$ est non dégénérée (car sa puissance extérieure maximale est une forme volume), nous en déduisons $\left.\left(\omega_{\varphi}^{\wedge a} \wedge \omega_{\psi}^{\operatorname{dim} Y-a}\right)\right|_{U} \neq 0$ d'où le résultat. 
Nous pouvons maintenant transformer la condition dans (2) à l'aide de dimensions. Pour lui donner une forme aussi proche que possible de notre objectif, nous posons pour $\varphi_{2} \in \operatorname{End}(A) \otimes \mathbb{R}$

$$
\mathcal{E}\left(\varphi_{2}\right)=\left\{\left(\varphi, \varphi_{1}\right) \in(\operatorname{End}(A) \otimes \mathbb{R})^{2} \mid \varphi^{*} \mathscr{L}=\varphi_{1}^{*} \mathscr{L}+\varphi_{2}^{*} \mathscr{L}, \operatorname{rg} \varphi \geq r \text { et } \varphi_{1} \neq 0\right\}
$$

et

$$
\mu\left(\varphi_{2}\right)= \begin{cases}0 & \operatorname{sir} \operatorname{rg} \varphi_{2} \geq r \\ \inf _{\left(\varphi, \varphi_{1}\right) \in \mathcal{E}\left(\varphi_{2}\right)} \operatorname{rg} \varphi_{1}-1 & \text { sinon. }\end{cases}
$$

Avec ces notations, nous avons la caractérisation suivante.

Lemme 4.7. Le fermé $Y$ est $r$-supertransverse si et seulement si pour tout $\psi \in$ $\operatorname{End}(A) \otimes \mathbb{R}$ on $a \operatorname{dim} \psi(Y) \geq \operatorname{dim} Y-\mu(\psi)$.

Démonstration. Notons d'abord que dans (2) $\operatorname{si} \operatorname{rg} \varphi \geq r$ nous pouvons toujours choisir $\varphi_{2}=\varphi, \varphi_{1}=0$ et $a=\operatorname{dim} Y$ de sorte que la condition contient en particulier $\left(\varphi^{*} \mathscr{L}\right)^{\cdot \operatorname{dim} Y} \cdot Y>0$ c'est-à-dire $\operatorname{dim} \varphi(Y)=\operatorname{dim} Y$ par le lemme précédent. Nous pouvons donc utiliser la seconde équivalence de ce même lemme pour dire que $Y$ est $r$-supertransverse si et seulement si

(3) pour tous $\varphi, \varphi_{1}, \varphi_{2} \in \operatorname{End}(A) \otimes \mathbb{R}$ et $a \in \mathbb{N}$ avec $\operatorname{rg} \varphi \geq r, \varphi^{*} \mathscr{L}=\varphi_{1}^{*} \mathscr{L}+\varphi_{2}^{*} \mathscr{L}$ et $\operatorname{rg} \varphi_{1}-1 \leq a \leq \operatorname{dim} Y$ nous avons $\operatorname{dim} \varphi_{2}(Y) \geq \operatorname{dim} Y-a$.

Par ailleurs affirmer $\operatorname{dim} \varphi_{2}(Y) \geq \operatorname{dim} Y-a$ pour tout $a \in \mathbb{N}$ avec $\operatorname{rg} \varphi_{1}-1 \leq a \leq$ $\operatorname{dim} Y$ revient simplement à $\operatorname{dim} \varphi_{2}(Y) \geq \operatorname{dim} Y-\max \left(0, \operatorname{rg} \varphi_{1}-1\right)$. Ensuite comme $\varphi_{1}=0$ ne peut survenir que lorsque $\operatorname{rg} \varphi_{2} \geq r$, la condition (3) est équivalente à

(4) pour tout $\varphi_{2} \in \operatorname{End}(A) \otimes \mathbb{R}$ avec $\operatorname{rg} \varphi_{2} \geq r$ on a $\operatorname{dim} \varphi_{2}(Y)=\operatorname{dim} Y$ et pour tout $\varphi_{2} \in \operatorname{End}(A) \otimes \mathbb{R}$ avec $\operatorname{rg} \varphi_{2}<r$ et tout $\left(\varphi, \varphi_{1}\right) \in \mathcal{E}\left(\varphi_{2}\right)$ on a $\operatorname{dim} \varphi_{2}(Y) \geq \operatorname{dim} Y-\left(\operatorname{rg} \varphi_{1}-1\right)$.

$\mathrm{Vu}$ la définition de $\mu$, ceci est exactement l'énoncé.

L'étape suivante consiste à reconnaître en $\mu$ la fonction $\mu_{\operatorname{End}(A) \otimes \mathbb{R}, r}$ annoncée plus haut. Pour cela, nous avons besoin de faire intervenir la structure d'anneau de $\operatorname{End}(A) \otimes \mathbb{R}$ et ceci nous amène à utiliser le lemme matriciel élémentaire suivant.

Lemme 4.8. Soient $n$ un entier, $\mathbb{K}$ l'un des trois corps $\mathbb{R}, \mathbb{C}$ ou $\mathbb{H}$ et $M, P \in \mathrm{M}_{n}(\mathbb{K})$ deux matrices telles que Ker $M \subset \operatorname{Ker} P$. Il existe $M^{\prime}, U, N \in \mathrm{M}_{n}(\mathbb{K})$ avec $N^{2}=$ $N, P N=0, M^{\prime}=U M, U$ inversible et ${ }^{t} \bar{M}^{\prime} M^{\prime}={ }^{t} \bar{N} N+{ }^{t} \bar{P} P$.

Démonstration. Nous notons $G$ le supplémentaire orthogonal de $\operatorname{Ker} M$ dans $\operatorname{Ker} P$ et choisissons pour $N$ la matrice de la projection $\mathbb{K}^{n} \rightarrow \mathbb{K}^{n}$ orthogonale sur $G$. Ainsi $N^{2}=N$ et ${ }^{t} \bar{N}=N$ sont clairs tout comme $P N=0$ car $\operatorname{Im} N=G \subset \operatorname{Ker} P$. 
Choisissons ensuite $Q$ telle que ${ }^{t} \bar{Q}=Q$ et $Q^{2}={ }^{t} \bar{P} P$ et posons $M^{\prime}=N+Q$. Il vient

$$
{ }^{t} \bar{M}^{\prime} M^{\prime}={ }^{t} \bar{N} N+{ }^{t} \bar{Q} Q+{ }^{t} \bar{N} Q+{ }^{t} \bar{Q} N={ }^{t} \bar{N} N+{ }^{t} \bar{P} P+N Q+Q N .
$$

Or ${ }^{t} \overline{Q N} Q N=N^{t} \bar{P} P N=0$ donc $Q N=0$ et ${ }^{t} \overline{N Q} N Q=Q N Q=0$ donc $N Q=0$. Enfin pour $X \in \mathbb{K}^{n}$ la formule obtenue montre

$$
\begin{aligned}
& M^{\prime} X=0 \Longleftrightarrow{ }^{t} \overline{M^{\prime} X} M^{\prime} X=0 \Longleftrightarrow{ }^{t} \overline{N X} N X+{ }^{t} \overline{P X} P X=0 \\
& \Longleftrightarrow N X=P X=0 \Longleftrightarrow X \in \operatorname{Ker} P \cap \operatorname{Ker} N \Longleftrightarrow X \in \text { Ker } M .
\end{aligned}
$$

Ainsi Ker $M^{\prime}=\operatorname{Ker} M$ et ceci montre l'existence de $U$.

Cet énoncé technique permet en fait de construire $\left(\varphi, \varphi_{1}\right) \in \mathcal{E}\left(\varphi_{2}\right)$ avec $\operatorname{rg} \varphi=$ $\operatorname{rg} \varphi_{1}+\operatorname{rg} \varphi_{2}$ et donc de simplifier la fonction $\mu$.

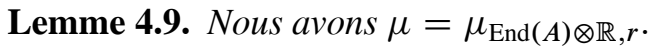

Démonstration. Pour abréger nous écrivons pour $\varphi_{2} \in \operatorname{End}(A) \otimes \mathbb{R}$

$$
\mathscr{F}\left(\varphi_{2}\right)=\left\{(\varphi, \psi) \in(\operatorname{End}(A) \otimes \mathbb{R}) \times(\operatorname{End}(V)) \mid \varphi_{2}=\psi \circ \varphi \text { et } \operatorname{rg} \varphi \geq r\right\} .
$$

L'examen des définitions de nos deux fonctions montre qu'il suffit d'établir, pour $\operatorname{rg} \varphi_{2}<r$,

$$
\inf _{\left(\varphi, \varphi_{1}\right) \in \mathcal{E}\left(\varphi_{2}\right)} \operatorname{rg} \varphi_{1}=\inf _{(\varphi, \psi) \in \mathcal{F}\left(\varphi_{2}\right)} \operatorname{rg} \varphi-\operatorname{rg} \varphi_{2} .
$$

Nous procédons par double inégalité. Considérons d'abord $\left(\varphi, \varphi_{1}\right) \in \mathcal{E}\left(\varphi_{2}\right)$ tel que $\operatorname{rg} \varphi_{1}$ soit minimal. Puisque $\varphi^{*} \mathscr{L}=\varphi_{1}^{*} \mathscr{L}+\varphi_{2}^{*} \mathscr{L}$ entraine $\operatorname{rg} \varphi \leq \operatorname{rg} \varphi_{1}+\operatorname{rg} \varphi_{2}$ nous avons $\operatorname{rg} \varphi_{1} \geq \operatorname{rg} \varphi-\operatorname{rg} \varphi_{2}$ et il suffit de vérifier qu'il existe $\psi$ avec $\varphi_{2}=\psi \circ \varphi$. Or l'égalité dans $\mathrm{NS}(A) \otimes \mathbb{R}$ se traduit par $H_{\varphi}=H_{\varphi_{1}}+H_{\varphi_{2}}$ donc pour $v \in V$

$$
v \in \operatorname{Ker} \varphi \Longleftrightarrow H_{\varphi}(v, v)=0 \Longleftrightarrow H_{\varphi_{1}}(v, v)=H_{\varphi_{2}}(v, v)=0 \Rightarrow v \in \operatorname{Ker} \varphi_{2}
$$

et $\operatorname{Ker} \varphi \subset \operatorname{Ker} \varphi_{2}$ nous donne bien l'existence de $\psi$.

Pour l'autre inégalité, nous considérons maintenant $(\varphi, \psi) \in \mathcal{F}\left(\varphi_{2}\right)$ avec $\operatorname{rg} \varphi$ minimal. La forme positive $H_{\psi}$ est dominée par un multiple de la forme définie positive $H$ donc il existe un réel $c>0$ tel que $c H_{\varphi}-H_{\varphi_{2}}$ soit une forme positive. Comme remplacer $\varphi$ par un multiple non nul ne change rien ici, nous supposons $c=1$. La positivité de $H_{\varphi}-H_{\varphi_{2}}$ signifie $\varphi^{*} \mathscr{L}-\varphi_{2}^{*} \mathscr{L} \in Z_{+}^{1}(A)$ donc il existe $\varphi_{1} \in \operatorname{End}(A) \otimes \mathbb{R}$ avec $\varphi^{*} \mathscr{L}=\varphi_{1}^{*} \mathscr{L}+\varphi_{2}^{*} \mathscr{L}$. Ceci ne suffit pas pour conclure car nous n'avons pas de contrôle sur le rang de $\varphi_{1}$. Mais $\operatorname{End}(A) \otimes \mathbb{R}$ est isomorphe à un produit d'anneaux de matrices sur $\mathbb{R}, \mathbb{C}$ ou $\mathbb{H}$ donc $\varphi, \varphi_{1}$ et $\varphi_{2}$ correspondent à des familles de matrices disons $\left(M_{i}\right),\left(N_{i}\right)$ et $\left(P_{i}\right)$. La relation $\varphi^{*} \mathscr{L}=\varphi_{1}^{*} \mathscr{L}+\varphi_{2}^{*} \mathscr{L}$ 
s'écrit ${ }^{t} \bar{M}_{i} M_{i}={ }^{t} \bar{N}_{i} N_{i}+{ }^{t} \bar{P}_{i} P_{i}$ (lorsque l'on choisit l'isomorphisme pour que l'involution de Rosati corresponde à la transconjugaison sur chaque facteur; voir [Mu, p. 208] et [R2, p. 343]) et implique $\operatorname{Ker} M_{i} \subset \operatorname{Ker} P_{i}$. Le lemme précédent fournit donc des matrices $M_{i}^{\prime}, U_{i}$ et $N_{i}^{\prime}$ avec $\left(N_{i}^{\prime}\right)^{2}=N_{i}^{\prime}, P_{i} N_{i}^{\prime}=0, M_{i}^{\prime}=U_{i} M_{i}$, $U_{i}$ inversible et ${ }^{t} \bar{M}_{i}^{\prime} M_{i}={ }^{t} \bar{N}_{i}^{\prime} N_{i}+{ }^{t} \bar{P}_{i} P_{i}$. En voyant les familles $\left(M_{i}^{\prime}\right),\left(U_{i}\right)$ et $\left(N_{i}^{\prime}\right)$ comme $\varphi^{\prime}, u, \varphi_{1}^{\prime} \in \operatorname{End}(A) \otimes \mathbb{R}$ nous avons $\varphi_{1}^{\prime} \circ \varphi_{1}^{\prime}=\varphi_{1}^{\prime}, \varphi_{2} \circ \varphi_{1}^{\prime}=0$, $\varphi^{\prime}=u \circ \varphi, u$ inversible et $\left(\varphi^{\prime}\right)^{*} \mathscr{L}=\left(\varphi_{1}^{\prime}\right)^{*} \mathscr{L}+\varphi_{2}^{*} \mathscr{L}$. Voyons que $\left(\varphi^{\prime}, \varphi_{1}^{\prime}\right) \in \mathcal{E}\left(\varphi_{2}\right)$ et $\operatorname{rg} \varphi_{1}^{\prime}=\operatorname{rg} \varphi-\operatorname{rg} \varphi_{2}$, ce qui donnera la conclusion.

Comme $u \in \operatorname{End}(V)$ est un automorphisme nous avons $\operatorname{rg} \varphi^{\prime}=\operatorname{rg} \varphi \geq r$. De plus $\varphi_{1}^{\prime}$ est non nul car sinon $\left(\varphi^{\prime}\right)^{*} \mathscr{L}=\varphi_{2}^{*} \mathscr{L}$ donne $\operatorname{rg} \varphi_{2}=\operatorname{rg} \varphi^{\prime}=\operatorname{rg} \varphi \geq r$ contrairement à notre hypothèse $\operatorname{rg} \varphi_{2}<r$. Ceci montre $\left(\varphi^{\prime}, \varphi_{1}^{\prime}\right) \in \mathcal{E}\left(\varphi_{2}\right)$. Comme plus haut, $H_{\varphi^{\prime}}=H_{\varphi_{1}^{\prime}}+H_{\varphi_{2}}$ donne $\operatorname{Ker} \varphi^{\prime}=\operatorname{Ker} \varphi_{1}^{\prime} \cap \operatorname{Ker} \varphi_{2}$. Enfin si $x \in V$ nous écrivons $x=\varphi_{1}^{\prime}(x)+\left(x-\varphi_{1}^{\prime}(x)\right)$ avec $\varphi_{1}^{\prime}(x) \in \operatorname{Ker} \varphi_{2} \operatorname{car} \varphi_{2} \circ \varphi_{1}^{\prime}=0$ et $x-\varphi_{1}^{\prime}(x) \in \operatorname{Ker} \varphi_{1}^{\prime} \operatorname{car} \varphi_{1}^{\prime} \circ \varphi_{1}^{\prime}=\varphi_{1}^{\prime}$. Ainsi $V=\operatorname{Ker} \varphi_{1}^{\prime}+\operatorname{Ker} \varphi_{2}$ de sorte que $\operatorname{Ker} \varphi^{\prime}=\operatorname{Ker} \varphi_{1}^{\prime} \cap \operatorname{Ker} \varphi_{2}$ donne bien $\operatorname{rg} \varphi^{\prime}=\operatorname{rg} \varphi_{1}^{\prime}+\operatorname{rg} \varphi_{2}$.

Si nous rassemblons les résultats obtenus jusqu'ici, nous voyons que $Z_{X}^{(r)}$ est l'union des fermés irréductibles $Y$ de $X$ pour lesquels il existe $\varphi \in \operatorname{End}(A) \otimes \mathbb{R}$ tel que $\operatorname{dim} \varphi(Y)<\operatorname{dim} Y-\mu_{\operatorname{End}(A) \otimes \mathbb{R}, r}(\varphi)$. Pour conclure, il nous reste essentiellement à voir que l'on peut remplacer $\varphi \in \operatorname{End}(A) \otimes \mathbb{R} \operatorname{par} \varphi \in \operatorname{End}(A)$ et c'est ici qu' intervient le théorème $\mathrm{d}^{\prime} \mathrm{Ax}$, sous la forme suivante.

Lemme 4.10. Soient $W$ un sous-espace vectoriel de $V, U$ un ouvert de A pour la topologie complexe, $Z$ une sous-variété analytique irréductible de $U$ et $Y$ l'adhérence de Zariski de $Z$ dans $A$. Si $\pi^{-1}(Z) \subset W+\Lambda$ il existe un sous-espace vectoriel $W^{\prime}$ de $V$ tel que $W \subset W^{\prime}, \pi^{-1}(Y) \subset W^{\prime}+\Lambda$ et $\operatorname{dim} W^{\prime} \leq \operatorname{dim} W+\operatorname{dim} Y-\operatorname{dim} Z$.

Démonstration. C'est le théorème 1 de $[\mathrm{Ax}]$ appliqué au groupe algébrique $A$ (noté $G$ ), au sous-groupe analytique connexe $\pi(W)$ (noté $A$ ) et à la sous-variété $Z$ (notée $K)$. Il fournit un sous-groupe analytique (noté $B$ ) que nous écrivons ici $\pi\left(W^{\prime}\right)$.

Finalement nous en déduisons le résultat cherché.

Théorème 4.1. Nous avons $Z_{X}^{(r)}=Z_{X}\left(\mu_{\operatorname{End}(A) \otimes \mathbb{R}, r)}\right.$.

Démonstration. Notons à nouveau $\mu=\mu_{\operatorname{End}(A) \otimes \mathbb{R}, r}$. L'inclusion $Z_{X}(\mu) \subset Z_{X}^{(r)}$ est claire : un fermé $Y \mu$-anormal vérifie la condition du lemme 4.7. Soit maintenant $x \in V$ tel que $\pi(x) \in Z_{X}^{(r)}$. Par définition de $\operatorname{dim} \varphi(\cdot)$, il existe $\varphi \in \operatorname{End}(A) \otimes \mathbb{R}$ tel que $\operatorname{dim}_{x}\left(\pi^{-1}(X) \cap(x+\operatorname{Ker} \varphi)\right)>\mu(\varphi)$. Nous considérons un voisinage ouvert $U$ de $x$ tel que $U \rightarrow \pi(U)$ est un isomorphisme puis une composante irréductible $Z$ de $\pi^{-1}(X) \cap(x+\operatorname{Ker} \varphi) \cap U$ contenant $x$ avec $\operatorname{dim} Z>\mu(\varphi)$. Soit $Y$ l'adhérence de $\pi(Z)$ dans $X$ pour la topologie de Zariski. Nous appliquons le lemme précédent à 
$W=\operatorname{Ker} \varphi, \pi(U-x), \pi(Z-x)$ et $Y-\pi(x)$. Il montre qu'il existe $W^{\prime}=\operatorname{Ker} \psi$ (où $\psi \in \operatorname{End}(V))$ tel que $W \subset W^{\prime}, \operatorname{dim} \psi(Y)=0$ et $\operatorname{rg} \psi \geq \operatorname{rg} \varphi+\operatorname{dim} Z-\operatorname{dim} Y>$ $\operatorname{rg} \varphi+\mu(\varphi)-\operatorname{dim} Y$. Par $(*)$ nous majorons $\operatorname{rg} \varphi+\mu(\varphi) \geq \operatorname{rg} \psi+\mu(\psi)$ (car $\operatorname{Ker} \psi \subset \operatorname{Ker} \varphi$ donne $\varphi=\chi \circ \psi$ et le lemme 4.1 autorise $\varphi \in \operatorname{End}(A) \otimes \mathbb{R})$ donc il vient $\operatorname{dim} Y>\mu(\psi)$. Le lemme 2.2 permet alors de remplacer $\psi$ par un élément de $\operatorname{End}(A)$ et donc $Y$ est $\mu$-anormal. Nous concluons $Y \subset Z_{X}(\mu)$ puis $\pi(x) \in Z_{X}(\mu)$ qui donne bien la seconde inclusion.

Le théorème 1.4 est maintenant entièrement démontré. Terminons en déduisant les théorèmes 1.5 et 1.3. Pour le premier, nous avons plus précisément l'énoncé suivant.

Proposition 4.2. Les assertions suivantes sont équivalentes.

(1) $Z_{X, \text { an }}^{(\operatorname{dim} X+1)} \neq X$;

(2) X est géométriquement non dégénérée;

(3) pour toute sous-variété abélienne $B$ de $A$ avec $\operatorname{deg} B \leq(\operatorname{deg} X)^{4^{g \operatorname{dim} X}}$ on $a$ :

$$
\operatorname{dim} X+B=\min (\operatorname{dim} X+\operatorname{dim} B, \operatorname{dim} A) .
$$

Démonstration. Écrivons $\mu=\mu_{\operatorname{End}(V), \operatorname{dim} X+1}$ puis pour $\psi \in \operatorname{End}(A)$ nous notons $Z_{X}(\mu, \psi)$ le fermé $\left\{x \in X \mid \operatorname{dim}_{x} X \cap \psi^{-1}(\psi(x))>\mu(\psi)\right\}$. Une égalité $Z_{X}(\mu, \psi)=X$ dit que la dimension de toutes les fibres de $X \rightarrow \psi(X)$ est au moins $\mu(\psi)+1$; elle signifie donc que la dimension relative de ce morphisme est au moins $\mu(\psi)+1$ c'est-à-dire $\operatorname{dim} \psi(X) \leq \operatorname{dim} X-\mu(\psi)-1$. Maintenant $\mu(\psi)=\max (0, \operatorname{dim} X-\operatorname{rg} \psi)$ d'où $\operatorname{dim} X-\mu(\psi)=\min (\operatorname{dim} X, \operatorname{rg} \psi)$. Enfin nous écrivons $B=\operatorname{Ker}^{0} \psi$ pour avoir $\operatorname{rg} \psi=g-\operatorname{dim} B$ et $\operatorname{dim} \psi(X)=$ $\operatorname{dim}(X+B) / B=\operatorname{dim}(X+B)-\operatorname{dim} B$. Par suite $Z_{X}(\mu, \psi)=X$ est équivalent à $: \operatorname{dim} X+B \leq \min (\operatorname{dim} X+\operatorname{dim} B, g)-1$.

Comme $Z_{X \text {,an }}^{(\operatorname{dim} X+1)}=Z_{X}(\mu)$ est l'union de tous les $Z_{X}(\mu, \psi)$ on constate facilement (1) $\Longrightarrow$ (2). Ensuite (2) $\Longrightarrow$ (3) est tautologique et (3) $\Longrightarrow$ (1) est conséquence du fait (voir partie 3) que $Z_{X}(\mu)$ est une union finie de $Z_{X}(\mu, \psi)$ pour $\psi \in \operatorname{End}(A)$ vérifiant $\operatorname{deg} \operatorname{Ker}^{0} \psi \leq(\operatorname{deg} X)^{q}$ où $q=p(p v+1)^{\operatorname{dim} X-1} \leq 4^{g \operatorname{dim} X}$.

Le résultat de [R1] combiné avec celui de [C] (qui démontre la conjecture 1.1 de [R1] dans le cas avec multiplications complexes) nous donne le fait suivant.

Proposition 4.3. Si A est à mutliplications complexes, pour tout choix de hauteur sur $A(\overline{\mathbb{Q}})$ et tout réel $H$ l'ensemble

$$
\left\{P \in\left(X(\overline{\mathbb{Q}}) \backslash Z_{X, \Gamma}^{(r)}\right) \cap\left(\Gamma+A^{[r]}\right) \mid H(P) \leq H\right\}
$$

est fini. 
Comme, de son côté, le théorème principal de [R2] montre que $\left(X(\overline{\mathbb{Q}}) \backslash Z_{X}^{(r)}\right) \cap$ $\left(\Gamma+A^{[r]}\right)$ est de hauteur bornée, on en déduit avec le théorème 1.4 que si $A$ est à multiplications complexes alors $\left(X(\overline{\mathbb{Q}}) \backslash Z_{X \text {,an }}^{(r)}\right) \cap\left(\Gamma+A^{[r]}\right)$ est fini et donc si $X$ est géométriquement non dégénéré il vient avec la proposition 4.2 que $X(\overline{\mathbb{Q}}) \cap(\Gamma+$ $A^{[\text {dim } X+1]}$ ) n'est pas dense dans $X: c^{\prime}$ 'est le théorème 1.3 .

\section{Exemples}

Nous illustrons brièvement dans cette partie le fait qu'aucune des inclusions $Z_{X, \mathbb{Q}}^{(r)} \subset Z_{X}^{(r)} \subset Z_{X \text {,an }}^{(r)}$ n'est en général une égalité. Cela nous permet aussi de présenter quelques calculs de fonctions $\mu$.

Nous nous plaçons dans le cas où $A=A_{0}^{2}$ avec $A_{0}$ une variété abélienne simple de dimension $n \geq 2$ (donc $g=2 n$ ). Nous considérons un fermé irréductible $Y_{1} \mathrm{de}$ $A$ de la forme $Y_{1}=Y_{0} \times\{0\}$ où $0 \in Y_{0} \subset A_{0}$ avec la condition $0<\operatorname{dim} Y_{0}<n$. Nous choisissons ensuite un sous-schéma fermé $X$ de $A$ de dimension $n$ contenant $Y_{1}$ mais différent de $A_{0} \times\{0\}$.

Nous remarquons que cette construction entraîne que $X$ n'est contenu dans aucun translaté de sous-variété abélienne stricte de $A$. En effet, pour une telle $B$, une inclusion $X \subset x+B$ impliquerait $X \subset B$ car $0 \in X$ et donc $Y_{1} \subset B$. Mais il est clair que seul $B=A_{0} \times\{0\}$ contient $Y_{1}$ donc nous aurions $X \subset A_{0} \times\{0\}$ puis par dimension $X=A_{0} \times\{0\}$, ce que nous avons exclu.

Évaluons maintenant les différentes fonctions $\mu$ pour $r=n+1$. Nous notons $\mu_{1}=\mu_{\operatorname{End}(A) \otimes \mathbb{Q}, r}$ et $\mu_{2}=\mu_{\operatorname{End}(V), r}$. Par la formule générale, nous avons $\mu_{2}(\varphi)=$ $\max (0, n-\operatorname{rg} \varphi)$. Maintenant $\operatorname{End}(A) \otimes \mathbb{Q}$ est de la forme $M_{2}(D)$ pour un certain corps $D$ et donc les éléments de $\operatorname{End}(A) \otimes \mathbb{Q}$ ont pour rang $0, n$ ou $2 n$. Par suite dans la définition de $\mu_{1}$ on ne peut donc choisir que $\operatorname{rg} \varphi^{\prime}=2 n$ et ceci conduit à la formule $\mu_{1}(\varphi)=\max (0,2 n-1-\operatorname{rg} \varphi)$. Autrement dit, pour $\varphi \in \operatorname{End}(A)$,

$$
\begin{array}{lll}
\text { si } \operatorname{rg} \varphi=2 n & \mu_{1}(\varphi)=0 & \mu_{2}(\varphi)=0, \\
\text { si } \operatorname{rg} \varphi=n & \mu_{1}(\varphi)=n-1 & \mu_{2}(\varphi)=0, \\
\text { si } \operatorname{rg} \varphi=0 & \mu_{1}(\varphi)=2 n-1 & \mu_{2}(\varphi)=n .
\end{array}
$$

Quels sont les fermés $\mu_{1}$-anormaux de $X$ ? La condition $\operatorname{dim} Y>\mu_{1}(\varphi)$ ne peut bien sûr avoir lieu pour $\mu_{1}(\varphi)=2 n-1$; lorsque $\mu_{1}(\varphi)=n-1$ elle entraîne $Y=X$ mais $X$ ne peut pas vérifier $\operatorname{dim} \varphi(X)=0 \operatorname{avec} \operatorname{rg} \varphi=n$ car $X$ n'est pas contenu dans un translaté de $\operatorname{Ker}^{0} \varphi$ par construction; enfin $\operatorname{si} \operatorname{rg} \varphi=2 n$ et $\operatorname{dim} Y>\mu_{1}(\varphi)=0$ on a $\operatorname{dim} \varphi(Y)=\operatorname{dim} Y>0$. Finalement $Z_{X, \mathbb{Q}}^{(r)}=Z_{X}\left(\mu_{1}\right)=\emptyset$.

Par ailleurs $Y_{1}$ est clairement $\mu_{2}$-anormal : en choisissant pour $\varphi: A_{0}^{2} \rightarrow A_{0}^{2}$ la projection sur le deuxième facteur, nous trouvons $\operatorname{dim} \varphi\left(Y_{1}\right)=0$ et $\operatorname{dim} Y_{1}>$ $\mu_{2}(\varphi)=0$. Ainsi $Z_{X \text {,an }}^{(r)}=Z_{X}\left(\mu_{2}\right) \neq \emptyset$. 
Reste à déterminer $Z_{X}^{(r)}$. Ici la réponse dépend de la nature de End $A_{0}$. Nous envisageons seulement deux cas extrêmes. Supposons tout d'abord End $A_{0}=\mathbb{Z}$ de sorte que $\operatorname{End}(A) \otimes \mathbb{R} \simeq \mathrm{M}_{2}(\mathbb{R})$. Dans ce cas, les éléments de $\operatorname{End}(A) \otimes \mathbb{R} \mathrm{n}$ 'ont eux aussi que trois rangs possibles : $0, n$ ou $2 n$. Par suite $\operatorname{rg} \varphi^{\prime}=2 n$ est également forcé dans la définition de $\mu_{\operatorname{End}(A) \otimes \mathbb{R}, r}$ donc cette fonction coïncide avec $\mu_{1}$. Ici $Z_{X, \mathbb{Q}}^{(r)}=Z_{X}^{(r)} \neq Z_{X, \text { an }}^{(r)}$.

Si au contraire $A_{0}$ est à multiplications complexes, End $\left(A_{0}\right) \otimes \mathbb{R} \simeq \mathbb{C}^{n}$ donc $\operatorname{End}(A) \otimes \mathbb{R} \simeq \mathrm{M}_{2}(\mathbb{C})^{n}$. On vérifie immédiatement que dans cet anneau (comme dans $\operatorname{End}(V) \simeq \mathrm{M}_{2 n}(\mathbb{C})$ ) pour tout élément $\varphi$ de rang au plus $r-1$ il existe un élément $\varphi^{\prime}$ de rang $r$ et une factorisation $\varphi=\psi \circ \varphi^{\prime}$. Par conséquent $\mu_{\operatorname{End}(A) \otimes \mathbb{R}, r}=\mu_{2}$ et nous avons cette fois-ci $Z_{X, \mathbb{Q}}^{(r)} \neq Z_{X}^{(r)}=Z_{X \text {,an }}^{(r)}$.

Ceci montre que les inclusions peuvent être strictes. Dans le sens opposé, nous avons égalité pour certaines classes de variétés abéliennes.

Lemme 5.1. Soient A une variété abélienne, $X$ un sous-schéma fermé intègre de $A$ et $r$ un entier avec $0 \leq r \leq g=\operatorname{dim} A$.

(1) Si A est isogène à un produit de courbes elliptiques alors $Z_{X, \mathbb{Q}}^{(r)}=Z_{X}^{(r)}=Z_{X \text {,an }}^{(r)}$.

(2) Si A est à multiplications complexes alors $Z_{X}^{(r)}=Z_{X \text {,an }}^{(r)}$.

Démonstration. Sous l'hypothèse de (1) l'égalité $Z_{X, \mathbb{Q}}^{(r)}=Z_{X \text {,an }}^{(r)}$ apparaît dans [R2, lemme 1.4]. Pour (2) la preuve est identique à celle faite ci-dessus dans la fin de l'exemple $: \operatorname{End}(A) \otimes \mathbb{R}$ est de la forme $\prod_{i=1}^{k} \mathrm{M}_{n_{i}}(\mathbb{C})$ avec $\sum_{i=1}^{k} n_{i}=g$ donc on peut toujours choisir $\operatorname{rg} \varphi^{\prime}=r$.

\section{Finitude et fermeture}

Dans cette partie, nous nous intéressons aux ensembles $Z_{X, \Gamma}^{(r)}$ et aux questions 1 et 2 de l'introduction. Comme plus haut, nous nous plaçons dans un cadre plus général. Pour une fonction $\mu: \operatorname{End}(V) \rightarrow \mathbb{N}$ et $S$ une partie quelconque de $A(\mathbb{C})$, nous disons qu'un fermé irréductible $Y$ est $S$ - $\mu$-anormal s'il existe $\varphi \in \operatorname{End}(A)$ vérifiant $\operatorname{dim} \varphi(Y)=0, \operatorname{dim} Y>\mu(\varphi)$ et $\varphi(Y) \in \varphi(S)$.

Ceci permet de reformuler la définition de $Z_{X, \Gamma}^{(r)}$ : c'est l'union des fermés $\Gamma_{\text {sat }}{ }^{-}$ $\mu_{\text {End }(V), r}$-anormaux de $X$ (voir introduction et [R1, page 531]). Pour $\Gamma=0$ nous avons $\Gamma_{\text {sat }}=A(\mathbb{C})_{\text {tors }}$ et nous retrouvons pour $r=\operatorname{dim} X+1$ la notion de fermé torsion-anormal décrite dans [BMZ] pour les tores. En outre la terminologie précédente de $\mu$-anormal se retrouve en prenant $S=A(\mathbb{C})$.

C'est un problème ouvert de montrer que l'union des fermés $S$ - $\mu$-anormaux de $X$ est un fermé mais nous pouvons tout de même montrer qu'un fermé $S$ - $\mu$-anormal 
maximal est de degré borné. Ceci fait l'objet du théorème ci-dessous que nous énoncerons après quelques compléments sur les fonctions $\mu$.

Dans la suite, nous supposerons comme dans la partie 3 que $\mu: \operatorname{End}(V) \rightarrow \mathbb{N}$ vérifie $(*)$ pour $(\varphi, \chi) \in \operatorname{End}(A) \times \operatorname{End}(V)$. Par conséquent nous pouvons associer à toute sous-variété abélienne $B$ de $A$ l'entier $\mu(B)$ tel que $\mu(B)=\mu(\varphi)$ pour tout $\varphi \in \operatorname{End}(A)$ avec $B=\operatorname{Ker}^{0} \varphi$. Avec cette notation (*) entrâne que si $B \subset B^{\prime}$ sont deux sous-variétés abéliennes de $A$ alors

$$
0 \leq \mu\left(B^{\prime}\right)-\mu(B) \leq \operatorname{dim} B^{\prime}-\operatorname{dim} B .
$$

Par ailleurs, en reformulant la définition ci-dessus, nous voyons que $Y$ est $S$ - $\mu$ anormal si et seulement s'il existe $s \in S$ et une sous-variété abélienne $B$ de $A$ avec $Y \subset s+B$ et $\operatorname{dim} Y>\mu(B)$.

Voyons maintenant comment restreindre une fonction $\mu$ à une sous-variété abélienne.

Lemme 6.1. Soient $A^{\prime}=V^{\prime} / V^{\prime} \cap \Lambda$ et $A^{\prime \prime}=V^{\prime \prime} / V^{\prime \prime} \cap \Lambda$ deux sous-variétés abéliennes de $A=V / \Lambda$ telles que $V^{\prime} \cap V^{\prime \prime}=0$ et $\mu: \operatorname{End}(V) \rightarrow \mathbb{N}$ une fonction vérifiant $(*)$ pour $(\varphi, \chi) \in \operatorname{End}(A) \times \operatorname{End}(V)$. Il existe une fonction $\mu^{\prime}: \operatorname{End}\left(V^{\prime}\right) \rightarrow \mathbb{N}$ vérifiant $(*)$ pour $(\varphi, \chi) \in \operatorname{End}\left(A^{\prime}\right) \times \operatorname{End}\left(V^{\prime}\right)$ de sorte que pour toute sous-variété abélienne $B$ de $A^{\prime}$ on ait $\mu^{\prime}(B)=\mu\left(B+A^{\prime \prime}\right)$.

Démonstration. Notons $W$ un supplémentaire de $V^{\prime} \oplus V^{\prime \prime}$ dans $V$ de sorte que $W / W \cap \Lambda$ soit une sous-variété abélienne de $A$. Nous choisissons $N \in \mathbb{N} \backslash\{0\}$ tel que $N \Lambda \subset V^{\prime} \cap \Lambda \oplus V^{\prime \prime} \cap \Lambda \oplus W \cap \Lambda$. Nous associons à $\varphi \in \operatorname{End}\left(V^{\prime}\right)$ le morphisme $N\left(\varphi \times 0 \times \operatorname{id}_{W}\right): V^{\prime} \times V^{\prime \prime} \times W \rightarrow V^{\prime} \times V^{\prime \prime} \times W$ vu comme élément de End $(V)$ et posons $\mu^{\prime}(\varphi)=\mu\left(N\left(\varphi \times 0 \times \operatorname{id}_{W}\right)\right)$. Si $\varphi \in \operatorname{End}\left(A^{\prime}\right)$ nous avons $N\left(\varphi \times 0 \times \operatorname{id}_{W}\right) \in \operatorname{End}(A)$ grâce à l'hypothèse sur $N$. De plus $\operatorname{Ker}(N(\varphi \times 0 \times$ id $))=\operatorname{Ker} \varphi+V^{\prime \prime}$ ce qui donne la formule $\mu^{\prime}(B)=\mu\left(B+A^{\prime \prime}\right)$. Enfin si $\chi \in \operatorname{End}\left(V^{\prime}\right)$ nous avons $\chi \times 0 \times \mathrm{id} \in \operatorname{End}(V)$ et $\left(\chi \times 0 \times \operatorname{id}_{W}\right) \circ N\left(\varphi \times 0 \times \operatorname{id}_{W}\right)=N\left(\chi \circ \varphi \times 0 \times \operatorname{id}_{W}\right)$. De cette façon, $(*)$ pour $\mu^{\prime}$ suit de $(*)$ pour $\mu$ avec la remarque $\operatorname{rg}\left(N\left(\varphi \times 0 \times \operatorname{id}_{W}\right)\right)=\operatorname{rg} \varphi+\operatorname{dim} W$.

Nous rappelons que des entiers $v$ et $p$ ont été associés à une variété abélienne $A$ (voir fin de la partie 2). Nous utiliserons ci-dessous $v_{B} \leq v_{A}$ et $p_{B} \leq p_{A}$ pour toute sous-variété abélienne $B$ de $A$.

Après cette remarque, notre résultat est le suivant.

Théorème 6.1. Pour toute variété abélienne polarisée $(A, \mathscr{L})$, toute partie $S$ de $A(\mathbb{C})$, tout sous-schéma fermé intègre $X$ de A, toute fonction $\mu: \operatorname{End}(A) \rightarrow \mathbb{N}$ satisfaisant (*) $\operatorname{pour}(\varphi, \chi) \in \operatorname{End}(A) \times \operatorname{End}(V)$ et tout fermé $S$ - $\mu$-anormal maximal $Y$ de $X$ on $a$

$$
\operatorname{deg} Y \leq(\operatorname{deg} X)^{(p v+1)^{\operatorname{dim} X(\operatorname{dim} X-1) / 2}} .
$$


Démonstration. Nous montrons l'énoncé par récurrence sur $\operatorname{dim} X$. Si $\operatorname{dim} X \leq 1$, nous avons $Y=X$ et le résultat est clair. Supposons donc $\operatorname{dim} X \geq 2$. Le fermé $Y$ est en particulier $\mu$-anormal donc contenu dans un fermé $Y_{1} \mu$-anormal maximal dans $X$. Par la proposition 3.2, nous avons $\operatorname{deg} Y_{1} \leq(\operatorname{deg} X)^{\alpha_{\operatorname{dim} X}}$ où $\alpha_{n}$ désigne $(p v+1)^{n-1}$. Si jamais $Y_{1} \neq X$ alors $Y$ est $S$ - $\mu$-anormal maximal dans $Y_{1}$ donc par hypothèse de récurrence $\operatorname{deg} Y \leq\left(\operatorname{deg} Y_{1}\right)^{\beta_{\operatorname{dim} Y_{1}}}$ où $\beta_{n}$ est ici $(p v+1)^{n(n-1) / 2}$. Nous en déduisons $\operatorname{deg} Y \leq(\operatorname{deg} X)^{\alpha_{\operatorname{dim} X} \beta_{\operatorname{dim} Y_{1}}}$. Comme $\beta_{\operatorname{dim} Y_{1}} \leq \beta_{\operatorname{dim} X-1}$ et $\alpha_{\operatorname{dim} X} \beta_{\operatorname{dim} X-1}=\beta_{\operatorname{dim} X}$ ceci donne $\operatorname{deg} Y \leq(\operatorname{deg} X)^{\beta_{\operatorname{dim} X}}$ qui est la formule cherchée.

Sinon nous avons $Y_{1}=X$ ce qui signifie qu'il existe une sous-variété abélienne $B$ de $A$ et $x \in A(\mathbb{C})$ avec $X \subset x+B$ et $\operatorname{dim} X>\mu(B)$. Il ne coûte rien de choisir $x \in Y$. En outre $X \subset x+B$ donne $\langle X\rangle \subset B$ donc par $(*) \mu(B) \geq \mu(\langle X\rangle)$ : ceci montre que nous pouvons supposer $B=\langle X\rangle$ sans rien changer. Maintenant l'hypothèse sur $Y$ donne l'existence d'une sous-variété abélienne $G$ de $A$ et de $s \in S$ avec $Y \subset s+G$ et $\operatorname{dim} Y>\mu(G)$. Puisque $x \in s+G$ nous avons $s+G=x+G$ donc $Y \subset X \cap(x+G) \subset(x+B) \cap(x+G)=x+(B \cap G)$. D'un autre côté $X \subset x+B \subset Y+B \subset s+G+B$. Nous examinons deux cas : $\operatorname{sid} \operatorname{dim} X>\mu(G+B)$ alors $X$ est $S$ - $\mu$-anormale donc $Y=X$ par maximalité et l'énoncé vaut ; sinon nous avons $\operatorname{dim} X \leq \mu(G+B)$.

Nous appliquons alors le lemme avec $A^{\prime}=B$. Pour $A^{\prime \prime}$ nous choisissons une sous-variété abélienne de $G$ telle que $G=(G \cap B)+A^{\prime \prime}$ et $A^{\prime \prime} \cap B$ fini. La fonction $\mu^{\prime}$ fournie par le lemme vérifie alors $\mu^{\prime}(G \cap B)=\mu(G)$ et $\mu^{\prime}(B)=\mu\left(B+A^{\prime \prime}\right)=$ $\mu(G+B)$. Écrivons $X^{\prime}=X-x$ et $Y^{\prime}=Y-x$ de sorte que $Y^{\prime} \subset X^{\prime} \subset B$. Par ce qui précède nous avons $Y^{\prime} \subset B \cap G$ puis $\operatorname{dim} Y^{\prime}>\mu^{\prime}(B \cap G)$ d'une part et $\operatorname{dim} X^{\prime} \leq \mu^{\prime}(B)$ d'autre part. Ainsi $Y^{\prime}$ est $\mu^{\prime}$-anormal (dans $B$ ) et $X^{\prime}$ ne l'est pas car $\left\langle X^{\prime}\right\rangle=B$ est la seule sous-variété abélienne de $B$ dont un translaté contient $X^{\prime}$.

Nous en déduisons que $Y^{\prime}$ est contenu dans un fermé $\mu^{\prime}$-anormal maximal $Y_{2}^{\prime} \neq$ $X^{\prime}$ de $X^{\prime}$. D'après la proposition 3.2 appliquée dans $B$ et grâce à la remarque sur $p$ et $v$, nous trouvons $\operatorname{deg} Y_{2}^{\prime} \leq\left(\operatorname{deg} X^{\prime}\right)^{\alpha_{\operatorname{dim} X^{\prime}}}$. En posant $Y_{2}=Y_{2}^{\prime}+x$ nous avons $\operatorname{deg} Y_{2}^{\prime}=\operatorname{deg} Y_{2}$ et $\operatorname{deg} X^{\prime}=\operatorname{deg} X$. Maintenant $Y$ est $S$ - $\mu$-anormal maximal dans $Y_{2}$ donc par récurrence $\operatorname{deg} Y \leq\left(\operatorname{deg} Y_{2}\right)^{\beta_{\operatorname{dim} Y_{2}}}$ et l'on conclut comme plus haut avec $Y_{1}$.

Pour alléger, nous appelons $\Gamma$-r-anormaux plutôt que $\Gamma_{\text {sat }}-\mu_{\operatorname{End}(V), r}$-anormaux les fermés intervenant dans la définition de $Z_{X, \Gamma}^{(r)}$. Voici comment la question 2 se réduit à la question 1 .

Théorème 6.2. Supposons que la question 1 admette une réponse positive dans tout quotient d'une variété abélienne A. Alors, pour tous $\Gamma$ et $r$, tout sous-schéma fermé intègre $X$ de A ne contient qu'un nombre fini de fermés $\Gamma$-r-anormaux maximaux et, en particulier, leur union $Z_{X, \Gamma}^{(r)}$ est un fermé. 
Démonstration. Nous procédons par récurrence sur $\operatorname{dim} X$, les cas où $\operatorname{dim} X \leq 1$ étant triviaux comme d'habitude. Comme un quotient d'un quotient de $A$ est un quotient de $A$, nous pouvons supposer que la conclusion du théorème vaut pour tout sous-schéma fermé intègre $X^{\prime}$ d'un quotient de $A$ avec $\operatorname{dim} X^{\prime} \leq \operatorname{dim} X-1$. Comme $\Gamma_{\text {sat }}$ est de rang fini, il ne coûte rien de supposer $\Gamma=\Gamma_{\text {sat }}$. Considérons donc un fermé irréductible $\Gamma$ - $r$-anormal maximal $Y$ de $X$. Il existe donc une sous-variété abélienne $G$ de $A$ et $\gamma \in \Gamma$ avec $Y \subset \gamma+G$ et $\operatorname{dim} Y>\max (0, r-1-\operatorname{codim} G)$. En outre d'après le théorème précédent $B=\langle Y\rangle$ appartient à un ensemble fini de sous-variétés abéliennes de $A$. Vu le résultat à montrer, nous pouvons supposer $B$ fixée. Notons $\varphi: A \rightarrow A / B$ le morphisme quotient et $y^{\prime}=\varphi(Y)$. Soit ensuite

$$
Z=\left\{x \in X \mid \operatorname{dim}_{x} X \cap \varphi^{-1}(\varphi(x)) \geq \operatorname{dim} Y\right\}
$$

qui est un fermé vérifiant $Y \subset Z \subset X$. De plus il y a un nombre fini de choix pour $Z$ donc pour ses composantes irréductibles. Si $Z \neq X$ nous pouvons appliquer l'hypothèse de récurrence à une telle composante $Z_{1}$ de $Z$ contenant $Y$ et dans laquelle $Y$ est évidemment encore $\Gamma$ - $r$-anormal maximal. Ceci montre que $Y$ appartient à un ensemble fini de fermés.

Examinons maintenant le cas où $Z=X$. Ceci signifie exactement que la dimension relative de $\left.\varphi\right|_{X}$ est au moins $\operatorname{dim} Y$ et implique en particulier que $\operatorname{dim} \varphi(X) \leq$ $\operatorname{dim} X-1(\operatorname{car} \operatorname{dim} Y>0)$. D'un autre côté $B \subset G \operatorname{donne} \operatorname{codim} \varphi(G)=\operatorname{codim} G \geq$ $r-\operatorname{dim} Y$ si bien qu'en notant $r^{\prime}=r-\operatorname{dim} Y$ nous avons $\varphi(G) \subset(A / B)^{\left[r^{\prime}\right]}$ et donc $y^{\prime} \in \varphi(\Gamma)+(A / B)^{\left[r^{\prime}\right]}$. Si nous écrivons

$$
y^{\prime} \in\left(\left(\varphi(X) \backslash Z_{\varphi(X), \varphi(\Gamma)}^{\left(r^{\prime}\right)}\right) \cap\left(\varphi(\Gamma)+(A / B)^{\left[r^{\prime}\right]}\right)\right) \cup Z_{\varphi(X), \varphi(\Gamma)}^{\left(r^{\prime}\right)},
$$

nous voyons qu'en vertu de la question 1 et de l'hypothèse de récurrence le membre de droite est un fermé $Z^{\prime}$ de $\varphi(X)$. Si $Z^{\prime} \neq \varphi(X)$ nous pouvons raisonner avec $X \cap \varphi^{-1}\left(Z^{\prime}\right)$ comme avec $Z$ ci-dessus et montrer que $Y$ appartient à une liste finie de fermés de $X$. Si $Z^{\prime}=\varphi(X)$ deux choses seulement peuvent se produire : ou bien $\varphi(X)$ est ponctuel ou bien il est $\varphi(\Gamma)-r^{\prime}$-anormal (notons ici que $\varphi(\Gamma)_{\text {sat }}=\varphi(\Gamma)$ ).

Si $\varphi(X)$ est ponctuel alors $X \subset Y+B \subset Y+G=\gamma+G$ donc $X$ est $\Gamma-r$ anormal et par maximalité de $Y$ nous avons $Y=X$. Ici la liste finie $\{X\}$ convient. Si $\varphi(X)$ est $\varphi(\Gamma)$ - $r^{\prime}$-anormal il existe une sous-variété abélienne $G^{\prime}$ de $A / B$ et $\gamma_{1} \in \Gamma$ tels que $\varphi(X) \subset \varphi\left(\gamma_{1}\right)+G^{\prime}$ et $\operatorname{dim} \varphi(X)>\max \left(0, r^{\prime}-1-\operatorname{codim} G^{\prime}\right)$. Notons $G_{1}=\varphi^{-1}\left(G^{\prime}\right)$ de sorte que $X \subset \gamma_{1}+G_{1}$ et codim $G_{1}=\operatorname{codim} G^{\prime}$. Par dimension relative, $\operatorname{dim} X \geq \operatorname{dim} \varphi(X)+\operatorname{dim} Y>\max \left(0, r-1-\operatorname{codim} G_{1}\right)$ donc $X$ est $\Gamma$ - $r$-anormal et à nouveau $Y=X$.

Remarque : dans [R1] (ici proposition 4.3) on peut en fait remplacer $Z_{X, \Gamma}^{(r)}$ par $Z_{X, \mathbb{Q}}^{(r)}$ voire $Z_{X, \mathbb{Q}, \Gamma}^{(r)}$ union des $\Gamma_{\text {sat }}-\mu_{\operatorname{End}(A), r}$-anormaux donc nos arguments montrent la finitude de $\left(X(\overline{\mathbb{Q}}) \backslash Z_{X}^{(r)}\right) \cap\left(\Gamma+A^{[r]}\right)$ si $A$ admet des multiplications complexes (ou vérifie la conjecture 1.1 de [R1]). 


\section{Références}

[Ax] J. Ax, Some topics in differential algebraic geometry. I. Analytic subgroups of algebraic groups. Amer. J. Math. 94 (1972), 1195-1204. Zbl 0258.14014 MR 0435088

[BMZ] E. Bombieri, D. Masser et U. Zannier, Anomalous subvarieties - structure theorems and applications. Internat. Math. Res. Notices 19 (2007), 1-33. Zbl 1145.11049 MR 2359537

[C] M. Carrizosa, Petits points et multiplication complexe. Internat. Math. Res. Notices 16 (2009), 3016-3097.

[D] O. Debarre, Fulton-Hansen and Barth-Lefschetz theorems for subvarieties of abelian varieties. J. Reine Angew. Math. 467 (1995), 187-197. Zbl 0833.14027 MR 1355928

[Hab] P. Habegger, Intersecting subvarieties of abelian varieties with algebraic subgroups of complementary dimension. Invent. Math. 176 (2009), 405-447. Zbl 05547480 MR 2495768

[Hoa] L. T. Hoa, Finiteness of Hilbert functions and bounds for Castelnuovo-Mumford regularity of initial ideals. Trans. Amer. Math. Soc. 360 (2008), 4519-4540. Zbl 1148.13009 MR 2403695

[Ma] G. Maurin, Courbes algébriques et équations multiplicatives. Math. Ann. 341 (2008), 789-824. Zbl 05294739 MR 2407327

[Mu] D. Mumford, Abelian varieties. Tata Institute of Fundamental Research Stud. Math. 5, Oxford University Press, London 1974. Zbl 0326.14012 MR 2514037

[P] R. Pink, A common generalization of the conjectures of André-Oort, Manin-Mumford, and Mordell-Lang. Prépublication 2005.

[Ran] Z. Ran, On subvarieties of abelian varieties. Invent. Math. 62 (1981), 459-479. Zbl 0474.14016 MR 0604839

[Rat] N. Ratazzi, Intersection de courbes et de sous-groupes et problèmes de minoration de hauteur dans les variétés abéliennes C.M. Ann. Inst. Fourier (Grenoble) 58 (2008), 1575-1633. Zbl 1156.11025 MR 2445828

[R1] G. Rémond, Intersection de sous-groupes et de sous-variétés I. Math. Ann. 333 (2005), 525-548. Zbl 1088.11047 MR 2198798

[R2] G. Rémond, Intersection de sous-groupes et de sous-variétés II. J. Inst. Math. Jussieu 6 (2007), 317-348. Zbl 05233246 MR 2311666

[Z] B. Zilber, Exponential sums equations and the Schanuel conjecture. J. London Math. Soc. (2) 65 (2002), 27-44. Zbl 1030.11073 MR 1875133

Received December 21, 2007

Gaël Rémond, Institut Fourier, BP 74, 38402 Saint-Martin-d'Hères Cedex, France

E-mail: Gael.Remond@ujf-grenoble.fr 